\title{
Factors Influencing the Development and Maintenance of Nocturnal Heavy-Rain-Producing Convective Systems in a Storm-Scale Ensemble
}

\author{
RUSS S. SCHUMACHER \\ Department of Atmospheric Sciences, Texas A\&M University, College Station, Texas, and Department of \\ Atmospheric Science, Colorado State University, Fort Collins, Colorado \\ ADAM J. CLARK \\ Cooperative Institute for Mesoscale Meteorological Studies, University of Oklahoma, and NOAA/OAR \\ National Severe Storms Laboratory, Norman, Oklahoma \\ Ming Xue And Fanyou Kong \\ Center for the Analysis and Prediction of Storms, University of Oklahoma, Norman, Oklahoma
}

(Manuscript received 17 August 2012, in final form 11 January 2013)

\begin{abstract}
From 9 to 11 June 2010, a mesoscale convective vortex (MCV) was associated with several periods of heavy rainfall that led to flash flooding. During the overnight hours, mesoscale convective systems (MCSs) developed that moved slowly and produced heavy rainfall over small areas in south-central Texas on 9 June, north Texas on 10 June, and western Arkansas on 11 June. In this study, forecasts of this event from the Center for the Analysis and Prediction of Storms' Storm-Scale Ensemble Forecast system are examined. This ensemble, with 26 members at 4-km horizontal grid spacing, included a few members that very accurately predicted the development, maintenance, and evolution of the heavy-rain-producing MCSs, along with a majority of members that had substantial errors in their precipitation forecasts. The processes favorable for the initiation, organization, and maintenance of these heavy-rain-producing MCSs are diagnosed by comparing ensemble members with accurate and inaccurate forecasts. Even within a synoptic environment known to be conducive to extreme local rainfall, there was considerable spread in the ensemble's rainfall predictions. Because all ensemble members included an anomalously moist environment, the precipitation predictions were insensitive to the atmospheric moisture. However, the development of heavy precipitation overnight was very sensitive to the intensity and evolution of convection the previous day. Convective influences on the strength of the MCV and its associated dome of cold air at low levels determined whether subsequent deep convection was initiated and maintained. In all, this ensemble provides quantitative and qualitative information about the mesoscale processes that are most favorable (or unfavorable) for localized extreme rainfall.
\end{abstract}

\section{Introduction}

Extreme precipitation, and the flooding and flash flooding it can cause, remains a particularly challenging prediction problem for numerical models and human forecasters (e.g., Fritsch and Carbone 2004; Novak et al. 2011). This stems in part from the small-scale, chaotic nature of the deep convection that is responsible for producing the heavy rainfall (e.g., Zhang et al. 2003) and

Corresponding author address: Russ Schumacher, Department of Atmospheric Science, Colorado State University, Fort Collins, CO 80523.

E-mail: russ.schumacher@colostate.edu the insufficient density of observations at the mesoscale (e.g., Dabberdt et al. 2005). Since deterministic predictions of heavy convective rainfall have limited skill, one promising technique for improving forecasts is ensemble prediction, whereby numerous forecasts are made with slight variations to the model initial conditions, the representation of subgrid-scale processes, the dynamics of the model itself, or some combination of these. Thus, ensembles allow for an estimate of the range of possible outcomes and quantification of the uncertainty in a given forecast. Ensembles can also be used to diagnose the dynamics of weather systems, by considering each member of the ensemble to be a different realization of a similar atmospheric state and contrasting the differences 
among the members (e.g., Hakim and Torn 2008). In this study, we will examine the utility of an ensemble of highresolution, convection-allowing forecasts for the analysis and prediction of an extreme rainfall event that occurred near a long-lived mesoscale convective vortex (MCV) in the southern United States.

\section{a. Extreme precipitation from MCVS}

One set of circumstances in which the ingredients for localized extreme precipitation (Doswell et al. 1996) can be brought together is when a midlatitude MCV persists for multiple diurnal cycles in a moist, weakly sheared environment. Raymond and Jiang (1990) showed that an $\mathrm{MCV}$, which is associated with a positive potential vorticity (PV) anomaly at midlevels, in an environment with ambient vertical wind shear will force ascent on its downshear side. Trier et al. (2000b) demonstrated that thermodynamic destabilization will also take place on the downshear flank of an MCV, as conditionally unstable air is lifted to saturation. Several detailed case studies have documented instances of heavy rainfall near MCVs in the United States (e.g., Bosart and Sanders 1981; Zhang and Fritsch 1987; Fritsch et al. 1994; Trier and Davis 2002; Nielsen-Gammon et al. 2005; Schumacher and Johnson 2008). In particular, Fritsch et al. (1994) showed how when the MCV's environment includes a low-level wind maximum and relatively weak winds aloft, the shear vector will oppose the midlevel flow, leading to persistent ascent near the MCV's center that results in slow-moving, heavy-rainproducing convection. Schumacher and Johnson (2009) summarized the common characteristics of extreme rainfall events occurring near MCVs, with an emphasis on the lifting and subsequent deep convection that occurs near the intersection of a low-level wind maximum and the midlevel vortex. Although these previous works have identified the processes that make some MCVs a favorable place for heavy-rain-producing convection to occur, it is not clear how often these synoptic and mesoscale conditions are in place but heavy rainfall does not occur. Or in other words, the conditions that are both necessary and sufficient for MCV-related extreme rainfall are not yet known. In this study, we will use high-resolution ensemble forecasts to begin to address these questions.

\section{b. Storm-scale ensemble prediction}

Ensemble forecasts have been used for nearly 20 years for operational global weather prediction models (e.g., Toth and Kalnay 1997; Buizza et al. 2005), and limitedarea ensembles such as the National Centers for Environmental Prediction (NCEP) Short-Range Ensemble Forecast system (SREF; Du et al. 1997) have been implemented at operational centers as well. However, until recently, it has only been practical to run ensembles in global or regional models with relatively coarse resolution, which is insufficient for skillfully predicting the mesoscale details of heavy rainfall. This gap has started to be closed in recent years by the experimental use of ensemble prediction systems in which a large number of limited-area models are run with sufficient resolution to explicitly predict convective motions. However, a stormscale ensemble requires a different design and configuration than a global ensemble or a coarse-resolution regional ensemble. In global ensemble prediction systems designed for medium-range prediction such as those run by the European Centre for Medium-Range Weather Forecasts (ECMWF; Buizza et al. 2005) and NCEP, initial condition perturbations are the primary source of diversity in the ensemble, as uncertainties in the initial conditions tend to produce larger errors than uncertainties in the model physics beyond about one day (e.g., Gilmour et al. 2001). For short-range predictions of convective weather, on the other hand, accounting for model errors and uncertainties in addition to those associated with the initial conditions becomes important as well (e.g., Stensrud et al. 2000; Eckel and Mass 2005).

One prominent effort in this area is the Storm-Scale Ensemble Forecast (SSEF) system that has been conducted in support of the National Oceanic and Atmospheric Administration (NOAA) Hazardous Weather Testbed (HWT) Spring Forecast Experiment (Clark et al. 2012a). Since 2007, the Center for the Analysis and Prediction of Storms (CAPS) has run a storm-scale ensemble during the HWT Spring Forecast Experiment, with an increased number of members at $4-\mathrm{km}$ horizontal grid spacing each year (Xue et al. 2007, 2011). Results from the SSEF for the prediction of severe weather and heavy rainfall have been very promising, as have the new methods for displaying and analyzing vast amounts of model output (e.g., Schwartz et al. 2010; Coniglio et al. 2010; Johnson et al. 2011; Clark et al. 2011, 2012a). The details of the 2010 SSEF will be presented in section 3. Other successful experiments using stormscale ensemble prediction have been shown for instances of deep convection and heavy precipitation in Europe (e.g., Hohenegger et al. 2008; Vié et al. 2011; Leoncini et al. 2013). Many questions remain about ensemble forecasting at convection-allowing resolutions, however, including the optimal design of such an ensemble and how to balance the size of the ensemble against the computational expense of running a large number of ensemble members at high resolution (e.g., Xue et al. 2011).

\section{c. Ensemble-based analysis of atmospheric processes}

In addition to improving weather forecasts, ensembles can be used for qualitative and quantitative analysis of weather systems. Hakim and Torn (2008) outlined several 

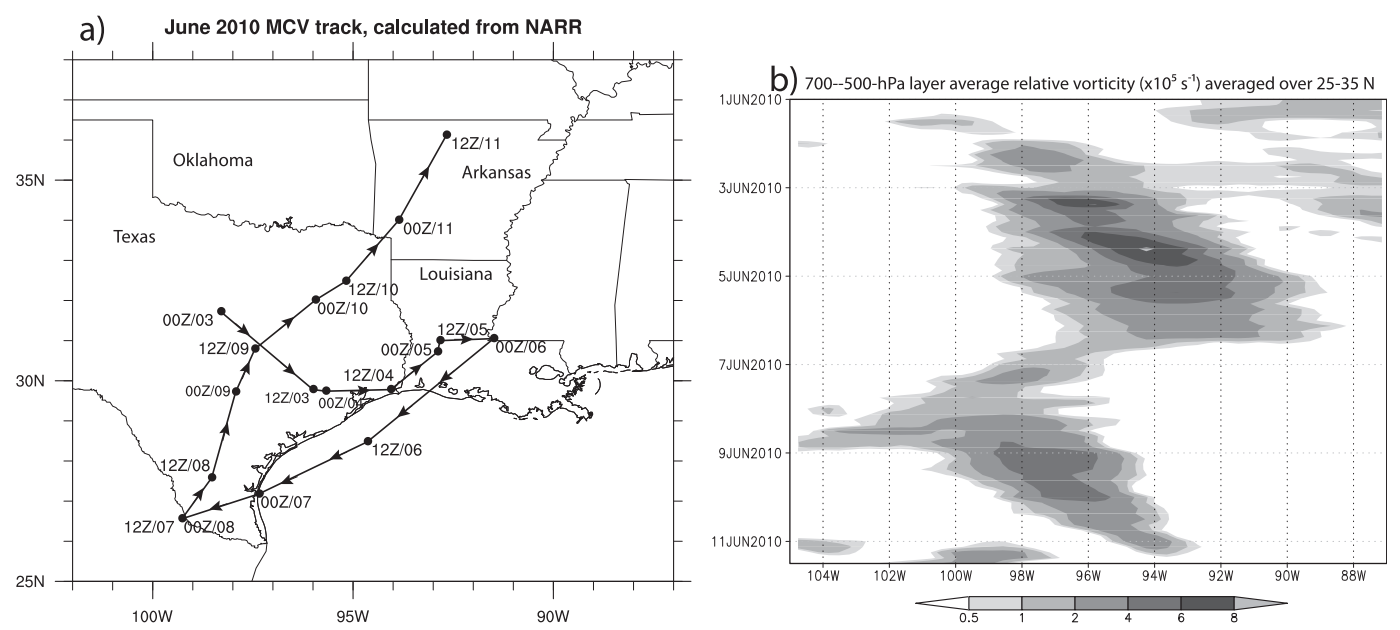

FIG. 1. (a) Objectively analyzed track of the long-lived MCV in June 2010. The track was determined by finding the $288 \mathrm{~km} \times 288 \mathrm{~km}(9 \times 9$ grid point $)$ box with the largest total 700-500-hPa layer-averaged relative vorticity in the NARR, and defining the center of that box as the MCV location. MCV locations are plotted every $12 \mathrm{~h}$, with the notation of, e.g., "00Z/03" indicating 0000 UTC 3 Jun 2010. Names of states mentioned in the text are also shown. (b) Timelongitude diagram of 700-500-hPa layer-averaged relative vorticity $\left(10^{5} \mathrm{~s}^{-1}\right)$, averaged over the $25^{\circ}-35^{\circ}$ latitude band.

methods by which an ensemble of forecasts can be used for diagnosing the dynamics of synoptic and mesoscale processes. In particular, calculating the covariance or correlation between a forecast metric of interest (such as the minimum pressure of a cyclone) and other meteorological fields can highlight important sensitivities in the evolution of that forecast metric. Martin and Xue (2006), Ancell and Hakim (2007), Hawblitzel et al. (2007), Sippel and Zhang $(2008,2010)$, and Torn (2010) all used these or similar methods to examine the dynamics and sensitivities of high-impact weather systems. Constructing subsets of members that have contrasting properties and analyzing the differences between those subsets can also reveal important information about the weather systems of interest; this method was used by Reinecke and Durran (2009), Schumacher (2011), and Hanley et al. (2011).

Herein, we will use a combination of manual and statistical diagnosis, along the same lines as the analysis of Clark et al. (2010b), to investigate the processes that were most favorable for, or detrimental to, the development of extreme precipitation in association with an MCV on 911 June 2010. Section 2 will provide an overview of this event, and section 3 will outline the data and methods used in the study. Section 4 will include an evaluation of the SSEF, along with the ensemble-based analysis of this event. A summary and conclusions are given in section 5 .

\section{Overview of the 9-11 June $2010 \mathrm{MCV}$ and heavy rainfall}

The MCV that ultimately led to the heavy rainfall from 9 to 11 June 2010 was exceptionally long lived. It originated from a squall line that developed in the Texas Panhandle late on 1 June 2010, with the first appearance of a circulation in radar reflectivity animations between 0600 and 1200 UTC 2 June (not shown; local standard time $=\mathrm{UTC}-6 \mathrm{~h}$ ). A strong MCV developed within the trailing stratiform region of a bow echo on 2-3 June, with a coherent circulation present in radar and satellite animations and in the North American Regional Reanalysis (NARR; Mesinger et al. 2006) midlevel vorticity field by 0000 UTC 3 June 2010 (Fig. 1). The MCV then moved southeastward (Fig. 1a) and intensified (Fig. 1b) on 4 June 2010. The vortex continued southeastward into south Texas and weakened from 5 to 7 June (Figs. 1a,b). Then, on 8 June, the MCV turned toward the north, moving into central Texas by 0000 UTC 9 June 2010 (Figs. 1a,b).

Between 0000 and 1200 UTC 9 June, the MCV intensified (Fig. 1b), was within an environment with a $15 \mathrm{~m} \mathrm{~s}^{-1}$ southerly low-level jet (LLJ; Fig. 2a), and was responsible in part for initiating and maintaining a small MCS over central Texas. This MCS produced locally extreme precipitation in and around San Antonio, Texas, with over $200 \mathrm{~mm}$ of rain falling in less than $6 \mathrm{~h}$ (Fig. 2b). Deadly flash flooding occurred on the Guadalupe River, with one fatality and over $\$ 10$ million in damage reported (NOAA 2012). The MCV intensified further in response to the latent heat release from this deep convection, and moved northeastward on 9-10 June (Figs. 1a,b and 2c). Another heavy-rain-producing MCS occurred from 0000 to 1500 UTC 10 June in northeast Texas (Fig. 2d), with over $200 \mathrm{~mm}$ of rain observed in some locations and damaging flooding reported in many parts of northeast 

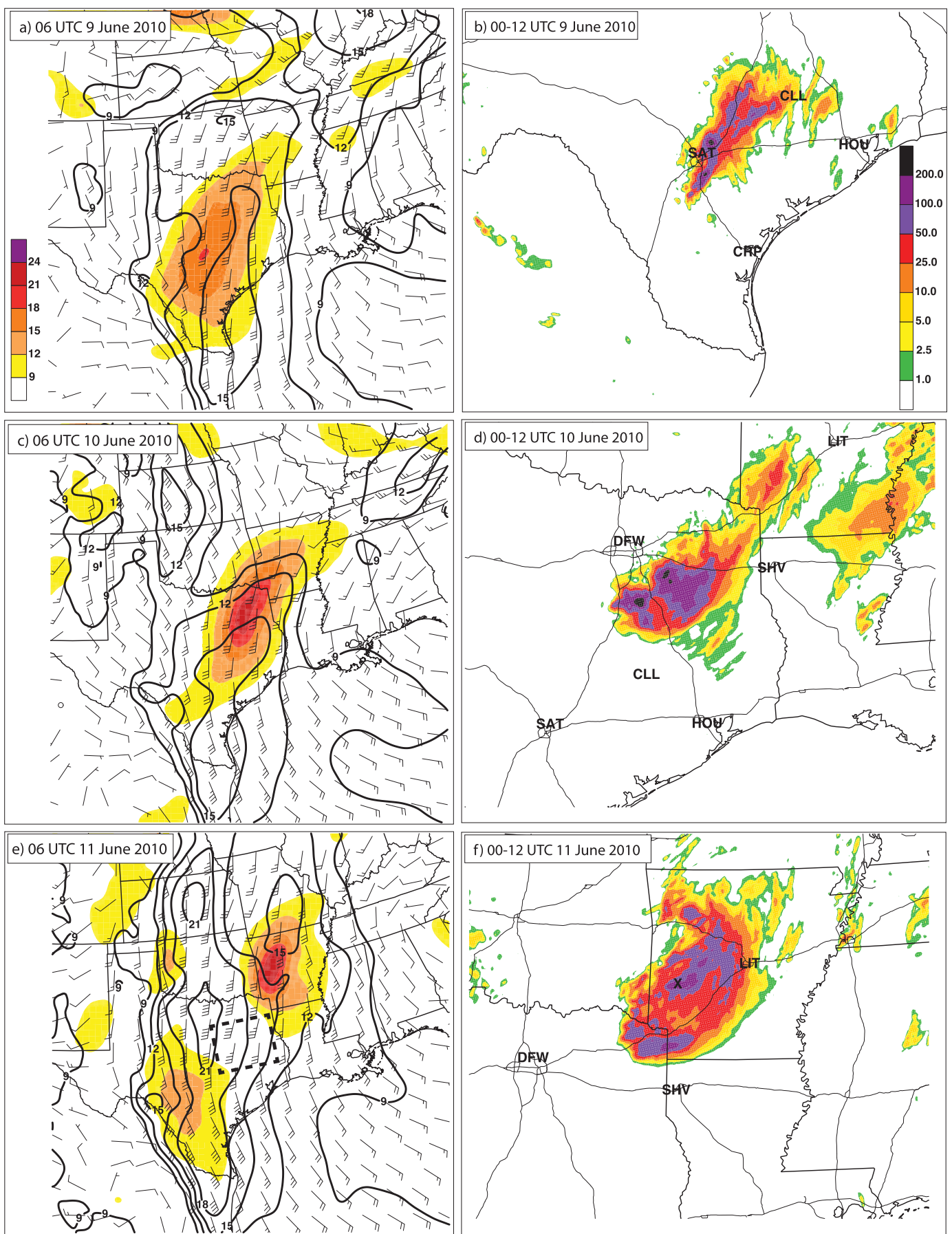

FIG. 2. (a),(c),(e) NARR 700-500-hPa layer-averaged absolute vorticity (color shading; $10^{5} \mathrm{~s}^{-1}$ ), $900-\mathrm{hPa}$ isotachs (contoured every $3 \mathrm{~m} \mathrm{~s}^{-1}$ starting at 9), and 900-hPa wind barbs (half barb $=5 \mathrm{kt}$; full barb $=10 \mathrm{kt}$; pennant $=50 \mathrm{kt}$; $1 \mathrm{kt}=0.5144 \mathrm{~m} \mathrm{~s}^{-1}$ ) at 0600 UTC (a) 9 Jun, (c) $10 \mathrm{Jun}$, and (e) 11 Jun 2010. (b),(d),(f) NCEP stage IV gridded precipitation analysis (mm; Lin and Mitchell 2005) for the 12-h periods ending (b) 9 Jun, (d) 10 Jun, and (f) 11 Jun 2010. In addition to state boundaries, interstate highways are drawn for reference, as are the locations of airports San Antonio (SAT), Houston Hobby (HOU), Dallas-Fort Worth (DFW), College Station (CLL), and Little Rock (LIT) and sounding locations Corpus Christi (CRP) and Shreveport (SHV). The location of the Albert Pike Recreation Area, where the deadly flash flood occurred on 11 Jun, is marked with an " $X$ " in (f). The area shown is the same for all three maps in the left column; the area shown changes from map to map in the right column. The dashed box in (e) shows the region where the area averages shown in Fig. 18 were calculated. 

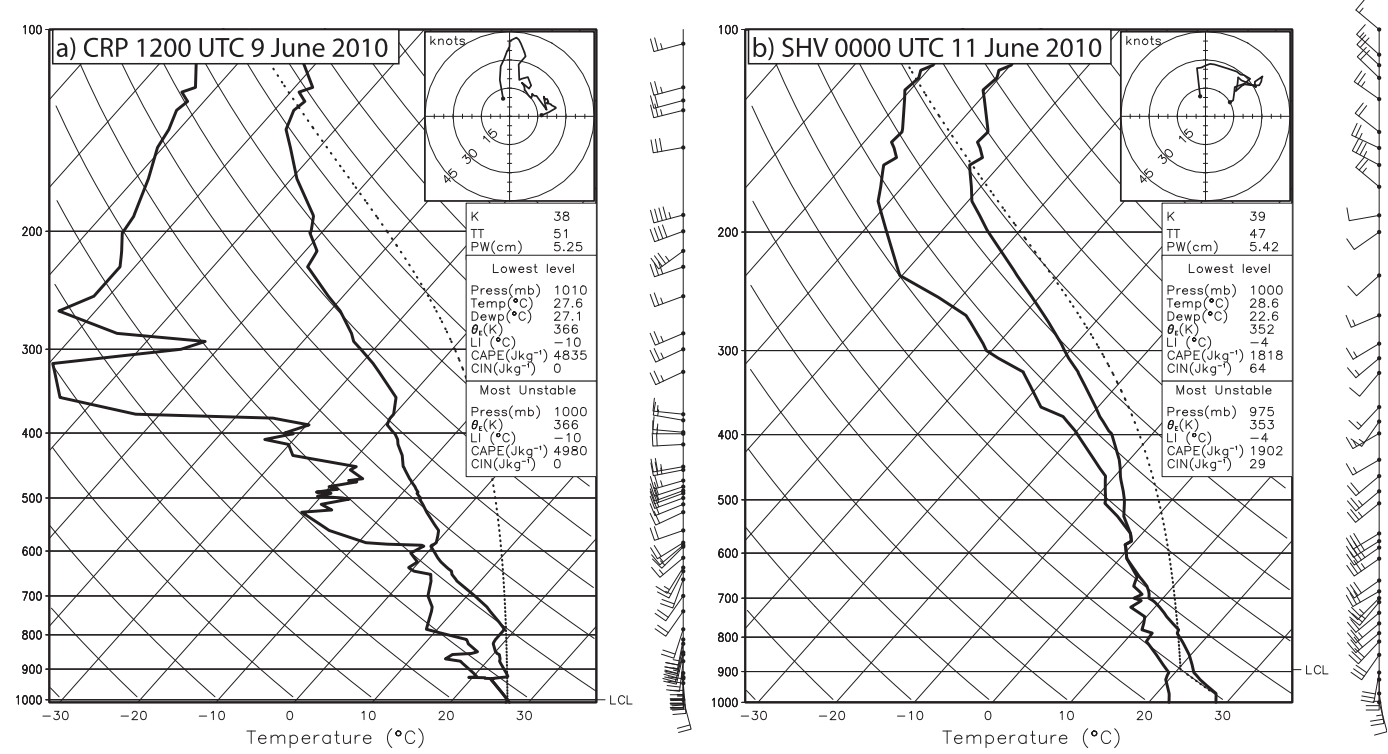

FIG. 3. Selected skew $T-\log p$ diagrams and wind hodographs from the inflow regions of heavy-rain-producing MCSs. (a) Corpus Christi, TX, at 1200 UTC 9 Jun 2010; and (b) Shreveport, LA, at 0000 UTC 11 Jun 2010. Parcel paths for the parcels with the highest $\theta_{e}$ in the lowest $3 \mathrm{~km}$ are shown by the dotted lines. The hodographs show the winds in the lowest $600 \mathrm{hPa}$ of the atmosphere. Locations of these soundings are shown in Figs. 2b,f.

Texas (NOAA 2012). This MCS invigorated the MCV and it continued moving toward the northeast (Figs. 1a,b and 2e). Once again an MCS developed during the evening and overnight hours of 11 June, with heavy precipitation falling over far northeastern Texas and western Arkansas (Fig. 2f). The maximum rainfall amounts on 11 June were slightly lower than on 9 and 10 June, but the rain fell on the complex terrain of western Arkansas and led to a deadly flash flood along the Little Missouri and Caddo Rivers. Twenty fatalities and 24 injuries occurred at the Albert Pike Recreational Area (indicated by the " $\mathrm{X}$ " in Fig. 2f) and damaging flash flooding was reported at other campgrounds in western Arkansas (NOAA 2012).

On each of these three nights, a heavy-rain-producing MCS developed when a southerly LLJ impinged upon an $\mathrm{MCV}$, consistent with the conceptual model proposed by Schumacher and Johnson (2009). Although it is difficult to identify radiosonde observations that are truly representative of the environments in which these mesoscale weather systems occurred, available soundings showed that these MCSs formed in moist and unstable conditions (Fig. 3). The Corpus Christi, Texas, sounding from 1200 UTC 9 June 2010 (Fig. 3a) was very unstable [convective available potential energy (CAPE) greater than $4500 \mathrm{~J} \mathrm{~kg}^{-1}$ ] and had a "hairpin" hodograph that can be favorable for slow-moving convection (e.g., Schumacher and Johnson 2009). The Shreveport, Louisiana, sounding from 0000 UTC 11 June 2010 was also unstable and was very moist, with precipitable water (PW) of $54 \mathrm{~mm}$ (Fig. 3b).

The analysis that follows will examine the skill and uncertainty of a convection-allowing ensemble in predicting these MCSs and their environment, along with the processes that were favorable for the development and maintenance of heavily precipitating convection.

\section{Data and methods}

\section{a. The SSEF}

Details of the 2010 version of the SSEF are given by Xue et al. (2010) and Clark et al. (2012b) and will not be repeated here, however, a brief summary is given below. In 2010, the SSEF was made up of 26 members at 4-km horizontal grid spacing that had diversity in initial and boundary conditions (ICs/LBCs), physical parameterizations, and the model dynamical cores. Three dynamical cores were used: the Advanced Research version of the Weather Research and Forecasting Model (ARW; Skamarock et al. 2008; 19 ensemble members), the Nonhydrostatic Mesoscale Model (NMM; Rogers et al. 2009; 5 members), and the Advanced Regional Prediction System (ARPS; Xue et al. 2003; 2 members). The IC and LBC diversity came from either assimilating or not assimilating radar observations, from perturbations derived from the Short-Range Ensemble Forecast (SREF; Du et al. 2006) system, and from other small-scale perturbations applied to three of the members (Xue et al. 
2010). Diversity in the physical parameterizations came from the use of different cloud microphysics, radiation, land surface, and planetary boundary layer schemes. The forecasts were initialized at 0000 UTC on weekdays from 17 May to 18 June 2010 and were integrated for $30 \mathrm{~h}$ over a domain that includes the conterminous United States. The configuration of each of the 26 ensemble members and the location of the model domain are given by Clark et al. (2012b).

\section{b. Observations and analyses}

Several observational and observation-based datasets will be used to evaluate the ensemble both in its forecasts of fields such as temperature and precipitation and in its representation of atmospheric processes. The NCEP stage IV precipitation analysis (Lin and Mitchell 2005), which uses radar-based precipitation estimates and adjusts them to ground-based gauges, will be used for evaluating precipitation forecasts. The NARR, along with radiosonde and wind profiler observations, will be used for examining upper-level atmospheric fields, and objectively analyzed surface observations (using the Barnes scheme; Koch et al. 1983) will be used for evaluating surface temperature forecasts. Composite radar reflectivity images from the National Mosaic and Multisensor Quantitative Precipitation Estimation (NMQ; Zhang et al. 2011) will be used to illustrate the radarobserved structures of precipitation systems.

\section{c. Ensemble-based analysis}

Many of the techniques that have been developed for ensemble-based synoptic and mesoscale analysis assume that all of the ensemble members are equally likely realizations of the atmosphere, and some also assume that linear statistics can be used to diagnose the differences between ensemble members. The first assumption does not strictly hold for the SSEF, as the configuration of the ensemble includes some members with only physics perturbations, some with only IC/BC perturbations, and some with both. ${ }^{1}$ Furthermore, the application of the perturbations is somewhat ad hoc, as the IC/LBC perturbations are taken from selected members of another

\footnotetext{
${ }^{1}$ As pointed out by Xue et al. (2010), some of the SSEF members were run for studying the impact of radar data assimilation and for examining the performance of particular physics parameterization schemes rather than for fully sampling forecast system uncertainties; these members are considered sensitivity forecast members. Only a subset of the 26 members have full IC/LBC and model perturbations and these were the members used for ensemble postprocessing for the real-time forecasting experiment. For studies such as this one, however, the sensitivity members are included and provide useful information.
}

ensemble with coarser grid spacing. Evaluation of the different ensemble members (discussed later in the manuscript) reveals that there is indeed a disparity in the characteristics of the members, with some having higher skill than others for heavy precipitation over the course of the experiment. For highly nonlinear processes such as deep convection, the assumption of linearity is also problematic. To mitigate the issues associated with these assumptions, we will manually analyze the evolution of individual members and subsets of members to diagnose their differences, and in particular we will investigate the differences between members that successfully predicted the timing and location of the heavy precipitation and those that did not. Linear statistics (such as correlation and covariance among members) will be used sparingly, and should be interpreted with some caution where they are used. For several of the predicted quantities from the ensemble, 5000-permutation bootstrap resampling tests were conducted to establish the statistical significance of differences between ensemble members. The confidence intervals derived from these tests are included where appropriate; however, these tests also revealed relationships that were statistically significant yet may not be physically significant, and therefore they are not strongly emphasized in the manuscript.

\section{d. Sensitivity experiments}

The SSEF system as it was run in real time provides a useful framework for investigating forecast skill and uncertainty as well as for diagnosing simulated atmospheric processes. However, because most of the ensemble members have more than one factor that differs from the other members (i.e., both the initial conditions and a parameterization are varied), it is not always possible to pinpoint the reasons for differences between members in a straightforward way. Therefore, several additional sensitivity simulations were conducted in which a single change was made to the configuration of a member and the differences analyzed. The details of these simulations and their results will be discussed in section $4 \mathrm{~d}$.

\section{Results}

\section{a. Evaluation of ensemble forecasts for 9-11 June 2010}

Before examining the mesoscale processes responsible for the heavy precipitation in more detail, an overview of the skill and uncertainty associated with SSEF precipitation forecasts during the 9-11 June 2010 period in comparison to the forecasts from the full 2010 HWT Spring Forecasting Experiment period is warranted. 

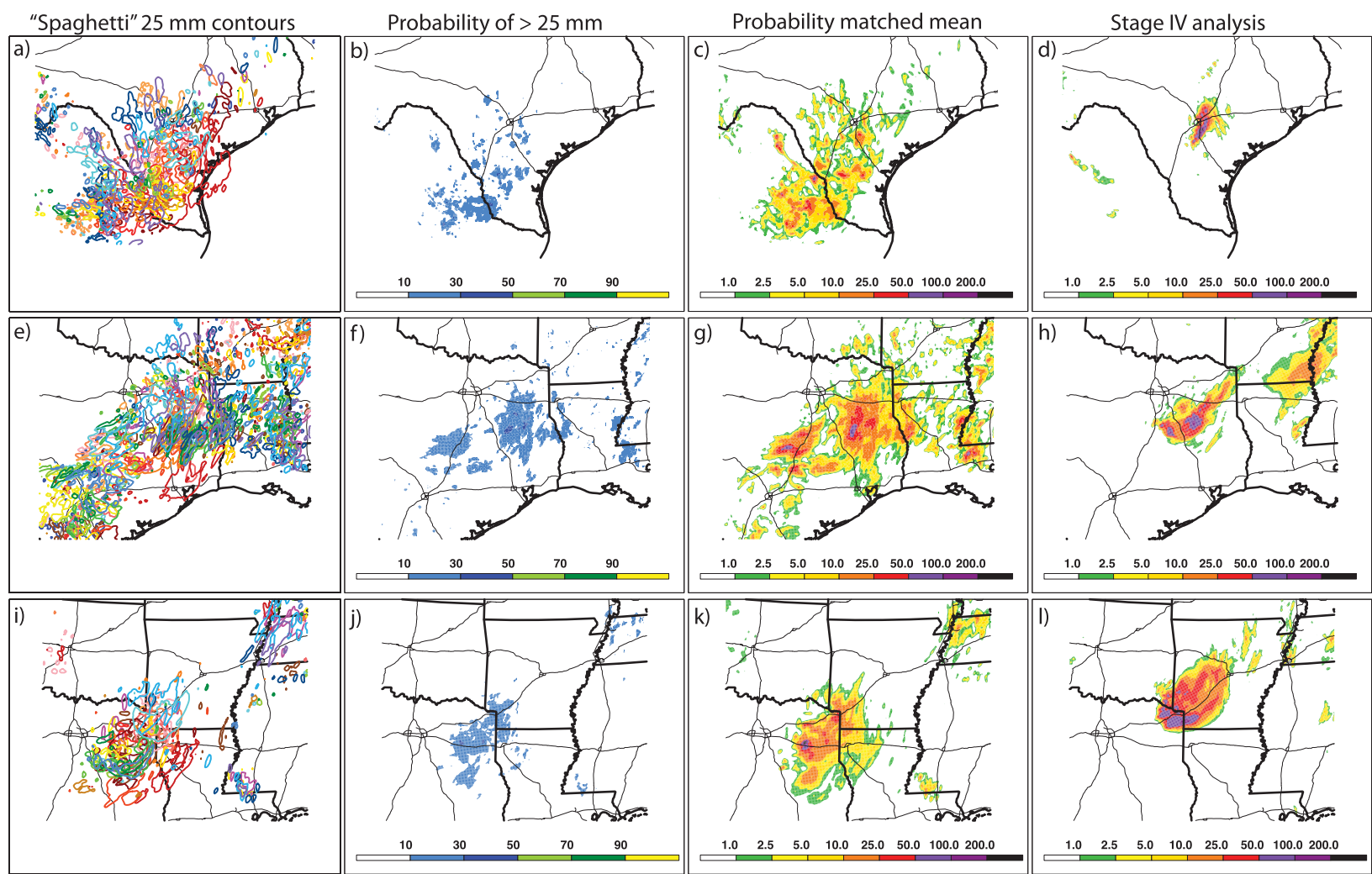

FIG. 4. SSEF 24-30-h precipitation forecasts valid for the 6-h periods ending 0600 UTC (a)-(c) 9 Jun, (e)-(g) 10 Jun, and (i)-(k) 11 Jun 2010, and stage IV precipitation analysis for the 6-h periods ending 0600 UTC (d) 9 Jun, (h) 10 Jun, and (l) 11 Jun 2010. (a),(e),(i) "Spaghetti" diagrams showing the predicted 25-mm precipitation contour from each ensemble member (different members are shown in different colors). (b),(f),(j) Ensemble probability (colors) of greater than $25 \mathrm{~mm}$ of precipitation. (c),(g),(k) Probability-matched mean precipitation $(\mathrm{mm})$.

For the analysis of 9-11 June, we focus on the 24-30-h forecasts for precipitation falling between 0000 and 0600 UTC. This timeframe is chosen because the heaviest precipitation during each of these three days occurred between 0000 and 1200 UTC, but the SSEF was only run out to $30 \mathrm{~h}$ in 2010 and therefore forecasts are only available through 0600 UTC. Furthermore, the computation time required for the SSEF means that it is more useful for "day 2" forecasts than for very-shortterm (i.e., $0-12 \mathrm{~h}$ ) forecasts.

Figure 4 shows three different methods for visualizing the ensemble output that were regularly used during the HWT Spring Forecast Experiment. The left column (Figs. $4 \mathrm{a}, \mathrm{e}, \mathrm{i}$ ) shows the forecast 25 -mm precipitation contour for each ensemble member in a different color, the second column from the left (Figs. $4 \mathrm{~b}, \mathrm{f}, \mathrm{j}$ ) shows the ensemble probability of $25 \mathrm{~mm}$ or more (where the probability is simply the percentage of ensemble members predicting that amount at each grid point), and the third column from the left (Figs. 4c,g,k) shows the probability-matched mean, which is intended to reflect the precipitation distribution associated with the ensemble mean but to retain the amplitude of the individual members (e.g., Ebert 2001; Clark et al. 2009). For each of the three nights, the SSEF had several members that predicted $25 \mathrm{~mm}$ of precipitation at or near the location where it was observed (Figs. 4d,h,l), but there was considerable spread in the forecast for the location of that precipitation (Figs. 4a,d,i). The probabilities of 12.5 (not shown), 25 (Figs. 4b,f,j), and $50 \mathrm{~mm}$ (not shown) were also relatively low in each of these forecasts in comparison to SSEF forecasts of other heavy precipitation events (e.g., Clark et al. 2012a, see their Fig. 6). Similarly, the probability-matched mean precipitation showed only isolated, if any, locations with $50 \mathrm{~mm}$ of precipitation or more (Figs. 4c,g,k), whereas this amount was observed on each of the three nights from 9 to 11 June (Figs. 4d,h,l). These forecasts are consistent with this time period having the possibility of heavy precipitation, but also having high uncertainty in whether, and where, it will occur. This uncertainty exists even within an environment that is known to be favorable for heavy rainfall, as all ensemble members have a long-lived MCV, deep moisture, and an LLJ, which will be shown in sections $4 \mathrm{~b}-\mathrm{d}$. 


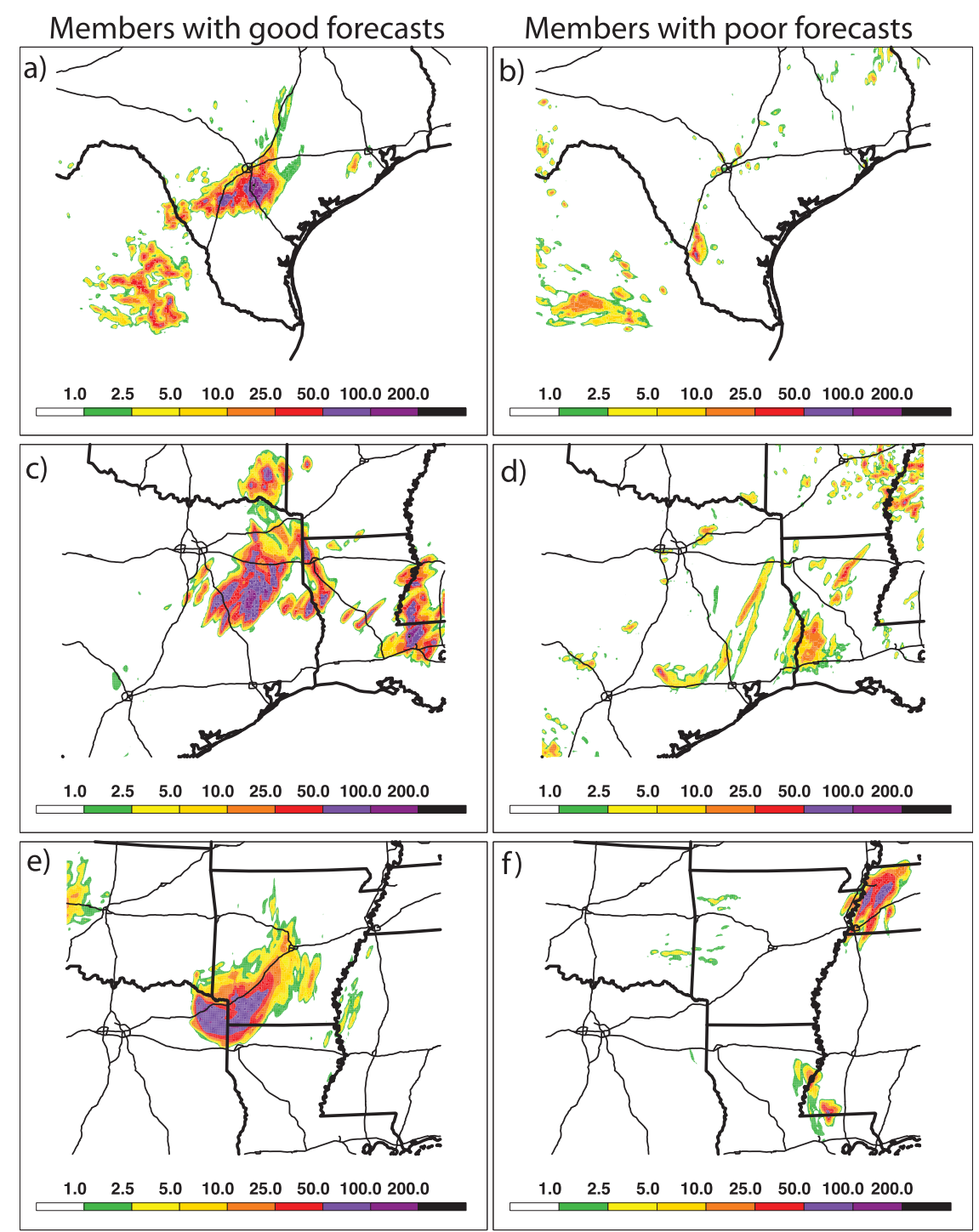

FIG. 5. Examples of ensemble members with good and poor forecasts of the heavy precipitation from 0000 to 0600 UTC (a),(b) 9 Jun; (c),(d) 10 Jun; and (e),(f) 11 Jun 2010. The maps shown and color scheme are as in Figs. 4d,h,l for comparison. Shown are (a) member arw_m9 and (b) member arw_m19 initialized 0000 UTC 8 Jun, (c) member nmm_m5 and (d) member arw_m15 initialized 0000 UTC 9 Jun, and (e) member arw_m5 and (f) member nmm_m5 initialized 0000 UTC 10 Jun. Configurations of the individual members are given in Clark et al. (2012b).

The diversity within the ensemble is also illustrated by comparing selected members that are representative of good and poor forecasts of the observed heavy precipitation (Fig. 5). For each of the heavy rainfall events on 9-11 June, there was at least one ensemble member that predicted approximately the correct location and intensity of the heavy precipitation (cf. Figs. 5a and 4d; Figs. $5 \mathrm{c}$ and $4 \mathrm{~h}$; Figs. 5e and 41 ), and some members that predicted near-zero rainfall in the 6-h period when heavy rain was observed (cf. Figs. 5b and 4d; Figs. 5d and 4h; Figs. $5 \mathrm{f}$ and 41 ). In the analysis to follow, these large differences between ensemble members with good and poor precipitation forecasts will be exploited to better understand the processes that are most important in initiating, organizing, and maintaining a heavy-rainproducing MCS.

The performance of the individual ensemble members over this three-day period can also be compared to their performance during the full 2010 experiment. Although a comprehensive evaluation of the 2010 SSEF is beyond the scope of this study, it is important to determine whether certain members consistently performed better 
or worse than others over the course of the experiment before more closely examining their forecasts of this event. Using the equitable threat score (ETS) with a 50-km neighborhood around each grid point, as described by Clark et al. (2010a), we find that all members produce skillful forecasts of $25.4 \mathrm{~mm}$ of precipitation in $6 \mathrm{~h}$. The ETS is highest at short lead times, decreasing to a minimum at hour 24 and then increasing slightly at hour 30 (Fig. 6a). The members using the NMM core generally have the lowest ETS. The forecasts over a subdomain including Texas, Oklahoma, Arkansas, and Louisiana initialized from 8 to 10 June 2010 (i.e., the forecasts of interest in this study), also show a minimum in skill at hour 24, with a few members performing well at hour 30 and others with near-zero ETS at hour 30 (Fig. 6b). Over the course of the experiment, most of the ARW members show a slight high bias at this rainfall threshold, with the NMM members exhibiting a substantially larger bias (Fig. 6c). Owing to this high bias in the NMM members, especially at longer lead times, these members will be excluded from some of the analyses shown in the manuscript. However, the finding that all of the ARW members had similar ETS and similar frequency biases for heavy rainfall at longer lead times provides support for giving these members equal consideration. Detailed descriptions of the performance of some of the individual members will be presented in the following section.

\section{b. Analysis of the 11 June 2010 MCS}

For the remainder of the manuscript, we will focus on the MCS that occurred between 0000 and 0600 UTC 11 June 2010 in far northeastern Texas and southwestern Arkansas (Fig. 4l). This is the 6-h period prior to when the Albert Pike campground flood took place. This specific time is chosen for several reasons. First, heavy precipitation was observed in association with an MCV, and therefore the scientific questions about the important processes in these situations can be addressed. Second, SSEF output for the 24-30-h forecast lead time is available for this time period. Third, there is adequate spread between ensemble members with very accurate precipitation forecasts and those with very poor forecasts (e.g., Figs. 5e,f) such that contrasting these sets of members will provide insights into the key processes for initiating and maintaining the MCS.

Although many of the ensemble members have their initial and lateral boundary conditions perturbed from the control analysis, the synoptic and mesoscale environment leading up to this time period was still very similar among all the members. All represented an MCV, a southerly LLJ, and a moist environment in their initial condition, and the motion of the MCV on 10 June was toward the northeast in all of the members. However,
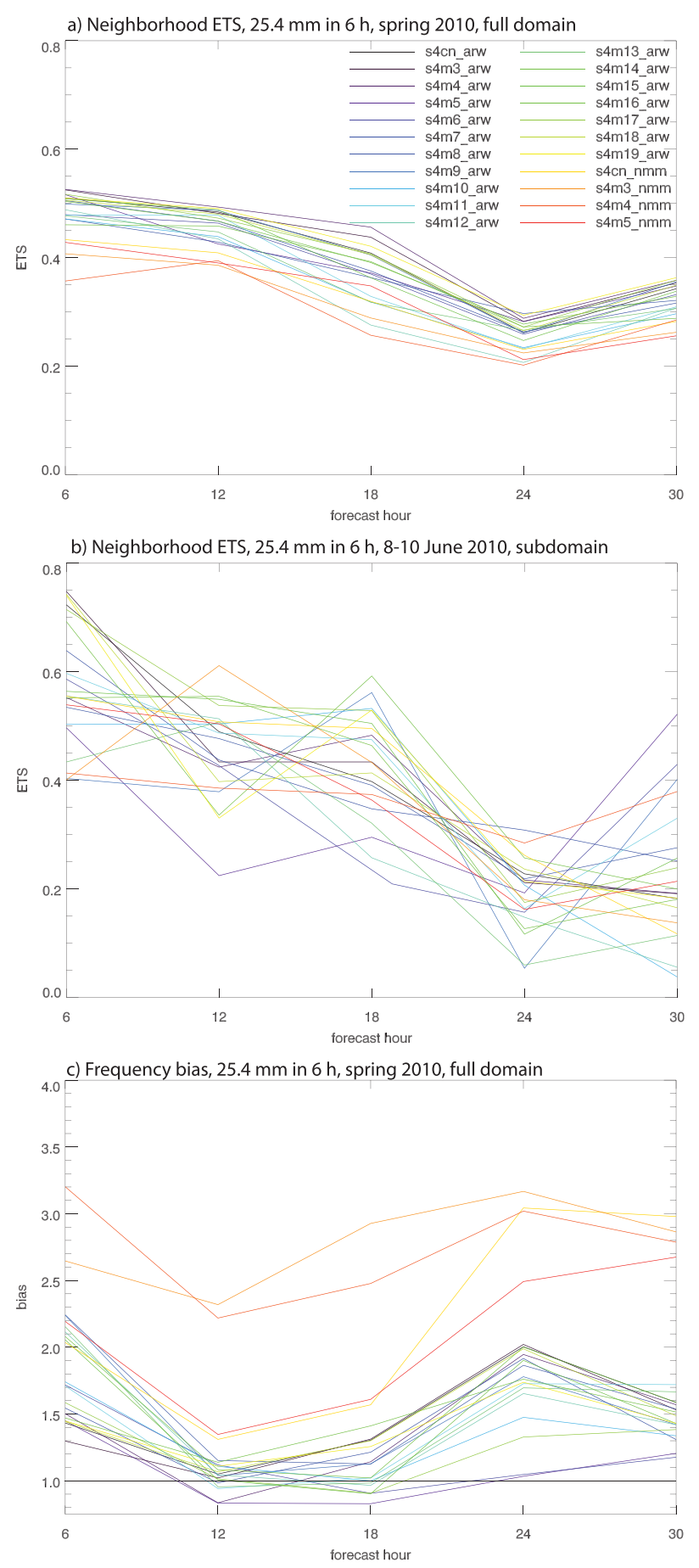

FIG. 6. (a) Equitable threat score (ETS) vs lead time for $25.4 \mathrm{~mm}$ of rain in 6 h, aggregated over the entire 2010 spring experiment. (b) As in (a), but only for the forecasts initialized 8-10 Jun 2010 over a subdomain including TX, OK, LA, and AR. Individual members are shown in different colors; ARPS members and those without radar data assimilation are excluded from the analysis. The ETS was calculated using a 50-km neighborhood around each grid point, as described by Clark et al. (2010a). Higher ETS indicates a better forecast, with 1 representing a perfect forecast and zero representing no skill. (c) As in (a), but for frequency bias. A frequency bias of 1 indicates that $25.4 \mathrm{~mm}$ of rain in $6 \mathrm{~h}$ was predicted the same number of times as it was observed; values above 1 indicate that this amount was predicted too frequently. 

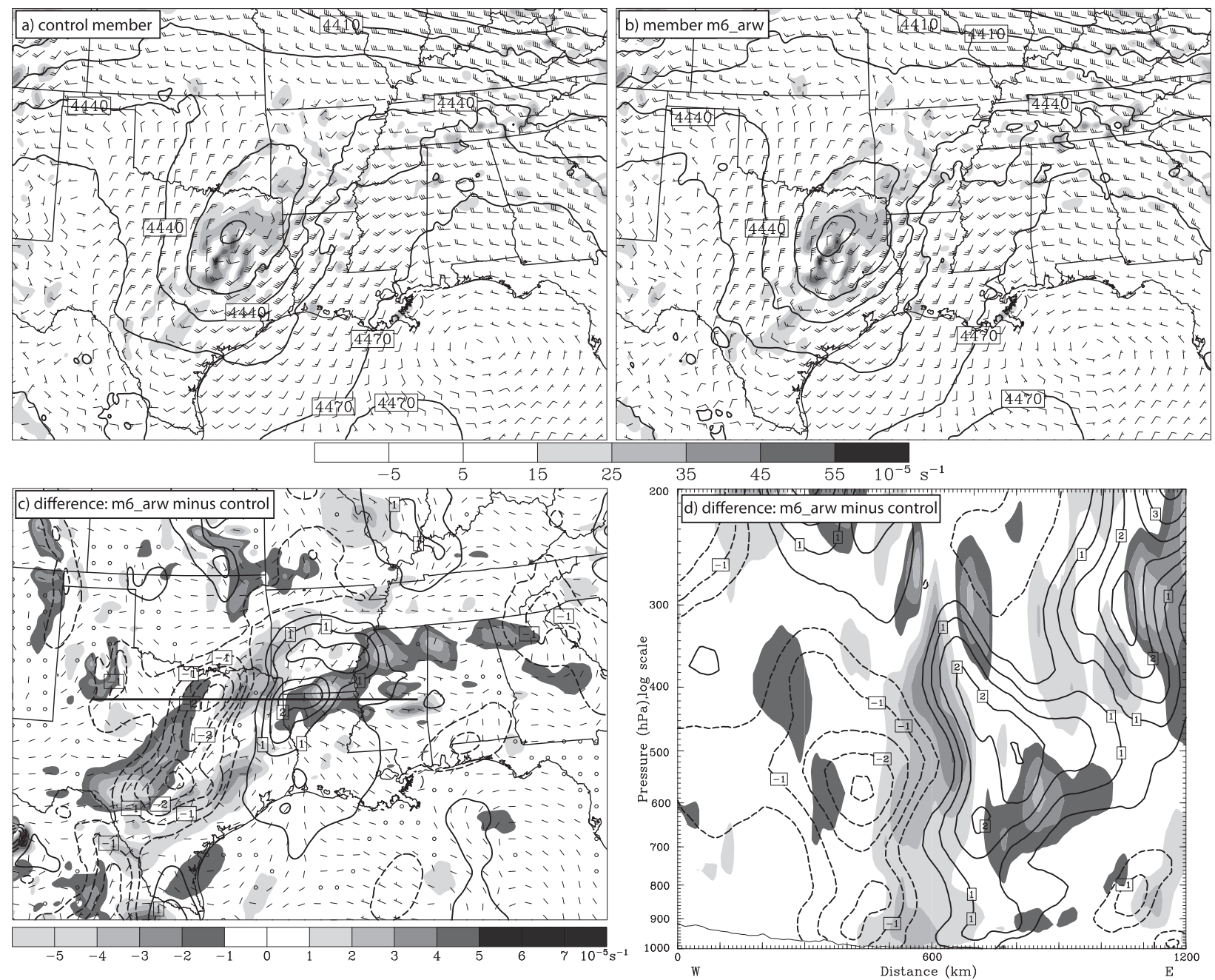

FIG. 7. (a) Absolute vorticity $\left(\times 10^{-5} \mathrm{~s}^{-1}\right.$, shaded), geopotential height ( $\mathrm{m}$, contoured), and winds (short barb represents $2.5 \mathrm{~m} \mathrm{~s}{ }^{-1}$, long barb represents $5 \mathrm{~m} \mathrm{~s}^{-1}$, pennant represents $25 \mathrm{~m} \mathrm{~s}^{-1}$ ) at $600 \mathrm{hPa}$ in the initial condition of the control member at 0000 UTC $10 \mathrm{Jun} 2010$. (b) As in (a), but for ensemble member m6_arw. (c) Difference fields between member m6_arw and the control member. Absolute vorticity differences $\left(\times 10^{-5} \mathrm{~s}^{-1}\right)$ are shaded and meridional wind speed differences are contoured every $0.5 \mathrm{~m} \mathrm{~s}^{-1}$, with negative contours dashed and the zero contour omitted. (d) As in (c), but for a vertical section along the west-east line shown in (c), and averaged over 20 grid points on either side of this line. The shading intervals are the same for (c) and (d).

in some members with perturbed ICs, the initial MCV was slightly stronger than in others. In particular, one of the IC perturbations, which was applied to members arw_m5 and arw_m6, produced a stronger vortex throughout the column than what was represented in the control member (Figs. 7c,d), although both of these members still had MCVs in approximately the same location (Figs. 7a,b). These two members also had differences in the placement of an elongated vorticity maximum that extended southwestward from the closed low through central and southern Texas (Fig. 7), although this feature dissipated in both members after forecast hour 18 , so this difference is likely not relevant to the development of precipitation the next night. The configurations of these members and their forecasts will be discussed in more detail shortly. Some members predicted the MCV to only progress into far northeastern Texas by 0600 UTC 11 June, whereas others predicted it to move into southwestern Arkansas, which was more consistent with observations (cf. Figs. 1a and 8). ${ }^{2}$

Considering the spread among the ensemble members shown in Figs. 4h-k, 5e,f, and 8, numerous characteristics

\footnotetext{
${ }^{2}$ When presenting upper-level fields, only the members using the ARW core are shown, because of limitations in obtaining, processing, and analyzing the full three-dimensional model output from the members using the other dynamical cores.
} 


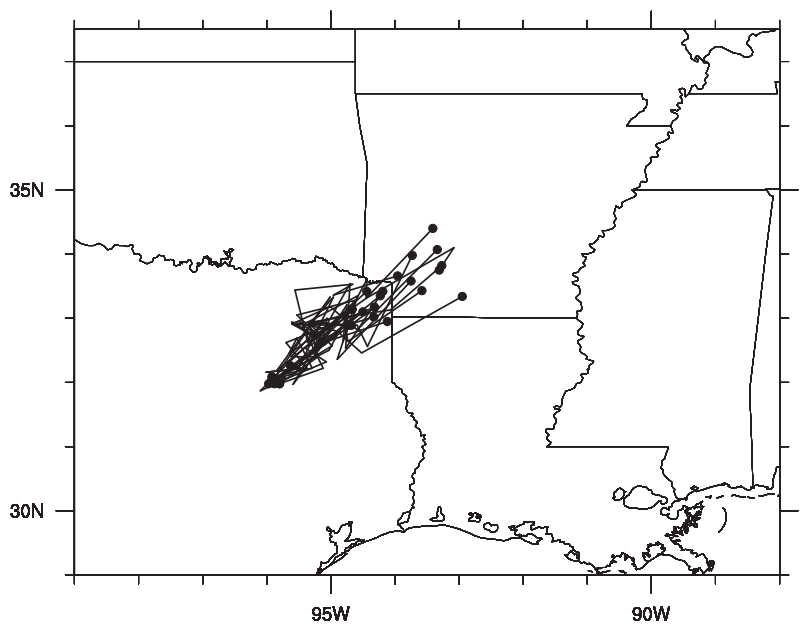

FIG. 8. As in Fig. 1a, but for the SSEF members between 0000 UTC 10 Jun and 0600 UTC 11 Jun 2010. Filled dots are shown for the MCV locations at 0600 UTC 11 Jun 2010. The method for objectively analyzing the MCV track is the same as that used in Fig. 1a, but the area of the box used for summation was $183 \mathrm{~km} \times$ $183 \mathrm{~km}(61 \times 61$ model grid points). Because of limitations in obtaining, processing, and analyzing three-dimensional model output, only the SSEF members using the ARW dynamical core are shown here.

of these members were analyzed to identify key differences between those correctly predicting heavy rainfall in northeastern Texas and southwestern Arkansas from 0000 to 0600 UTC 11 June. This analysis included manual inspection of surface and upper-air fields thought to be potentially important in the initiation and maintenance of this MCS, including the location of surface boundaries, the amount of atmospheric moisture and instability available, the strength of the MCV and the LLJ, and so on. Based on this analysis, several hypotheses for why the heavy rain occurred in some members but not others were developed and tested using the SSEF.

The primary hypothesis gleaned from this analysis was that the persistence of deep convection on the morning of 10 June, which influenced the intensity of the MCV and a near-surface dome of cold air, was related to the subsequent initiation, organization, and maintenance of the extreme-rain-producing MCS on 11 June. To illustrate the evidence in support of this hypothesis but to avoid overwhelming the reader with many multiplepaneled figures, three subsets of members (described in Table 1) will be presented that are representative of the behavior of larger groups of members, with more general results encompassing the entire ensemble shown as appropriate. One of these subsets (members arw_cn and arw_c0) represents the control configuration of the SSEF, with the only difference between the two being that radar data were assimilated into the ICs for member arw_cn and they were not for member arw_c0. The second subset (members arw_m5 and arw_m6) had perturbed ICs and LBCs, and also had different physics parameterizations than the control. These members used the Morrison, rather than the Thompson, microphysics scheme; the Yonsei University (YSU) rather than the Mellor-Yamada-Janjić (MYJ) PBL scheme, and the Rapid Update Cycle (RUC) instead of the Noah land surface model. As will be shown below, these members provided the best forecasts of the 11 June 2010 MCS. The third subset used the control ICs and LBCs, but used different microphysics parameterization: member arw_m15 used the WRF double-moment 6-class microphysics scheme (WDM6) and member arw_m16 the WRF single-moment 6-class microphysics scheme (WSM6). The discussion to follow will demonstrate that these members represented a convectively generated cold pool differently from the other subsets.

The first evidence in support of this hypothesis using the ensemble subsets is presented in Fig. 9. Although nearly all of the ensemble members correctly predicted an MCS in north Texas during the first $12 \mathrm{~h}$ of their forecast (not shown), the time at which that MCS dissipated differed among the ensemble members (Fig. 9).

TABLE 1. Differences in the configurations of the subset of SSEF members shown in Figs. 9. "Radar" refers to whether or not radar data were assimilated into the ICs. Microphysics schemes include Thompson et al. (2008); Morrison et al. (2005); WRF single-moment 6-class microphysics scheme (WSM6; Hong and Lim 2006) and WRF double-moment 6-class microphysics scheme (WDM6; Lim and Hong 2010). Land surface models include the Noah (Chen and Dudhia 2001) and Rapid Update Cycle (RUC; Smirnova et al. 2000). Planetary boundary layer (PBL) parameterizations include Mellor-Yamada-Janjić (MYJ; Mellor and Yamada 1982; Janjić 2002) and Yonsei University (YSU; Noh et al. 2003). Further details about these and the other SSEF members are given by Clark et al. (2012b), and technical descriptions of the parameterizations are given by Skamarock et al. (2008).

\begin{tabular}{lllllr}
\hline \hline Member & \multicolumn{1}{c}{ ICs } & LBCs & Radar & Microphysics & Land surface \\
\hline Arw_cn & Control & Control & Yes & Thompson & Noah \\
Arw_c0 & Control & Control & No & Thompson & Noah \\
Arw_m5 & + em-p1 & em-p1 & Yes & Morrison & RUC \\
Arw_m6 & + em-p1 recursive & em-p1 & Yes & Morrison & RU \\
Arw_m15 & Control & Control & Yes & WDM6 & Noah \\
Arw_m16 & Control & Control & Yes & WSM6 & YSU \\
\hline
\end{tabular}



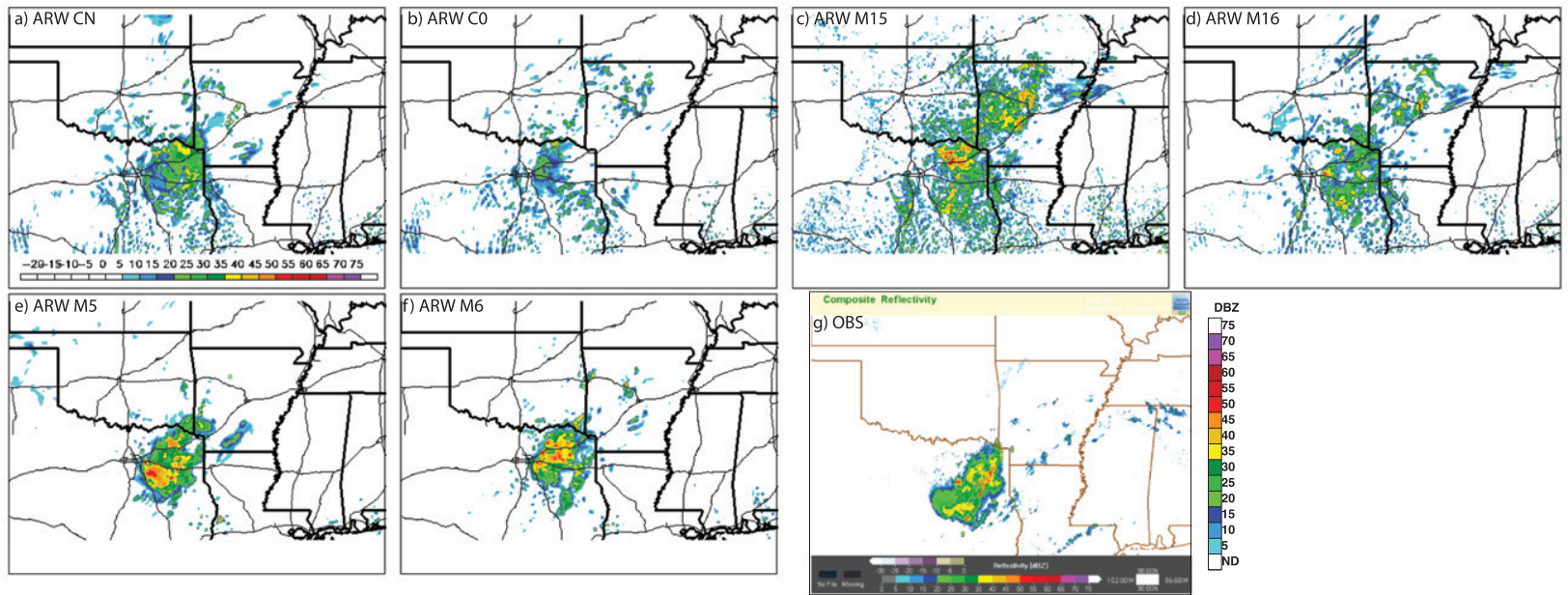

FIG. 9. (a)-(f) Simulated composite radar reflectivity (dBZ) from selected SSEF members initialized at 0000 UTC 10 Jun 2010 and valid at 1500 UTC10 Jun 2010. Members shown are (a) arw_cn, (b) arw_c0, (c) arw_m15, (d) arw_m16, (e) arw_m5, and (f) arw_m6. Differences in the configurations of these members are shown in Table 1. (g) Observed composite radar reflectivity at 1500 UTC 10 Jun 2010. Image obtained from the National Mosaic and Multisensor QPE (NMQ) website.

Radar observations at 1500 UTC 10 June indicated that the MCS was weakening, but still had some embedded convection within a larger area of stratiform precipitation (Fig. 9g). Some of the SSEF members had simulated radar reflectivity fields that were very similar to the observations at 1500 UTC (Figs. 9c,e,f), whereas others predicted the convection to have dissipated by this time (Figs. 9a,b,d).

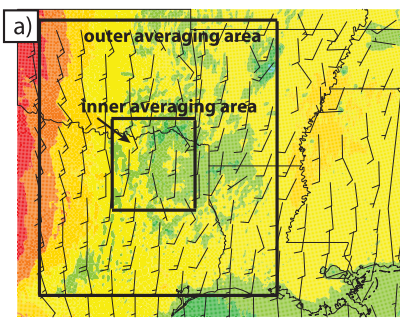

MEMBER S4CN ARW VALID 100610/1800V018 291294297300303306309312315318321
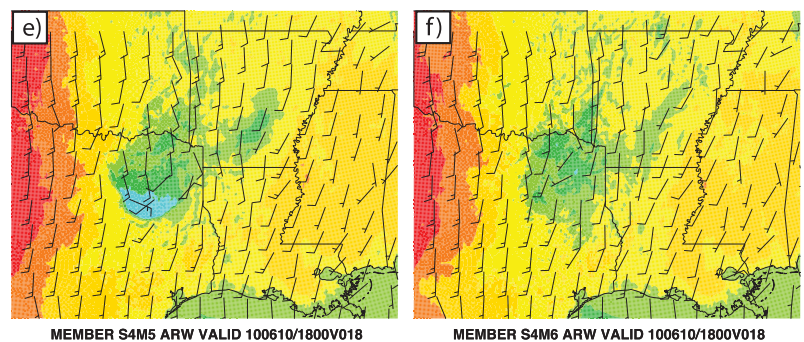

In observations and in the ensemble members that predicted the 10 June MCS to persist through the morning, a region of cool air was present in the low levels of the atmosphere in north Texas (Fig. 10g). This dome of cool air owed its existence to latent cooling from the earlier convection and reduced insolation from cloud cover (e.g., Trier et al. 2000a). A dome of cool air is commonly observed beneath mature MCVs during the daytime (e.g.,
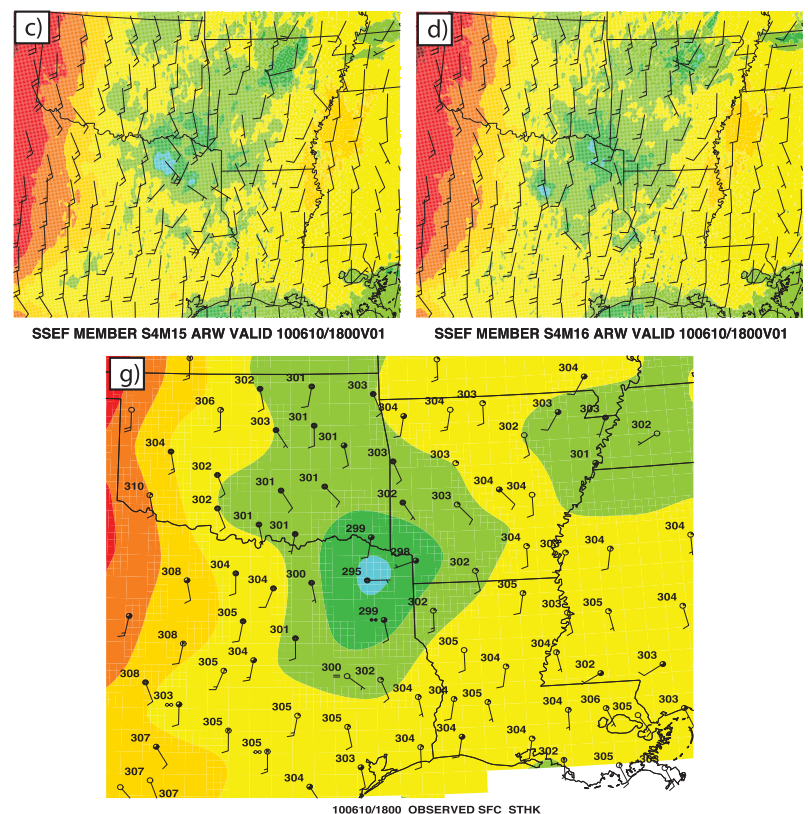

FIG. 10. (a)-(f) As in Fig. 9, but for potential temperature (K) at $2 \mathrm{~m}$ AGL and winds at $10 \mathrm{~m}$ AGL, valid at 1800 UTC 10 Jun 2010 . The rectangles in (a) show where the area-averaged potential temperature in Fig. 14 was calculated. (g) Objectively analyzed surface observations using the Barnes scheme (Koch et al. 1983) at 1800 UTC 10 Jun 2010. 

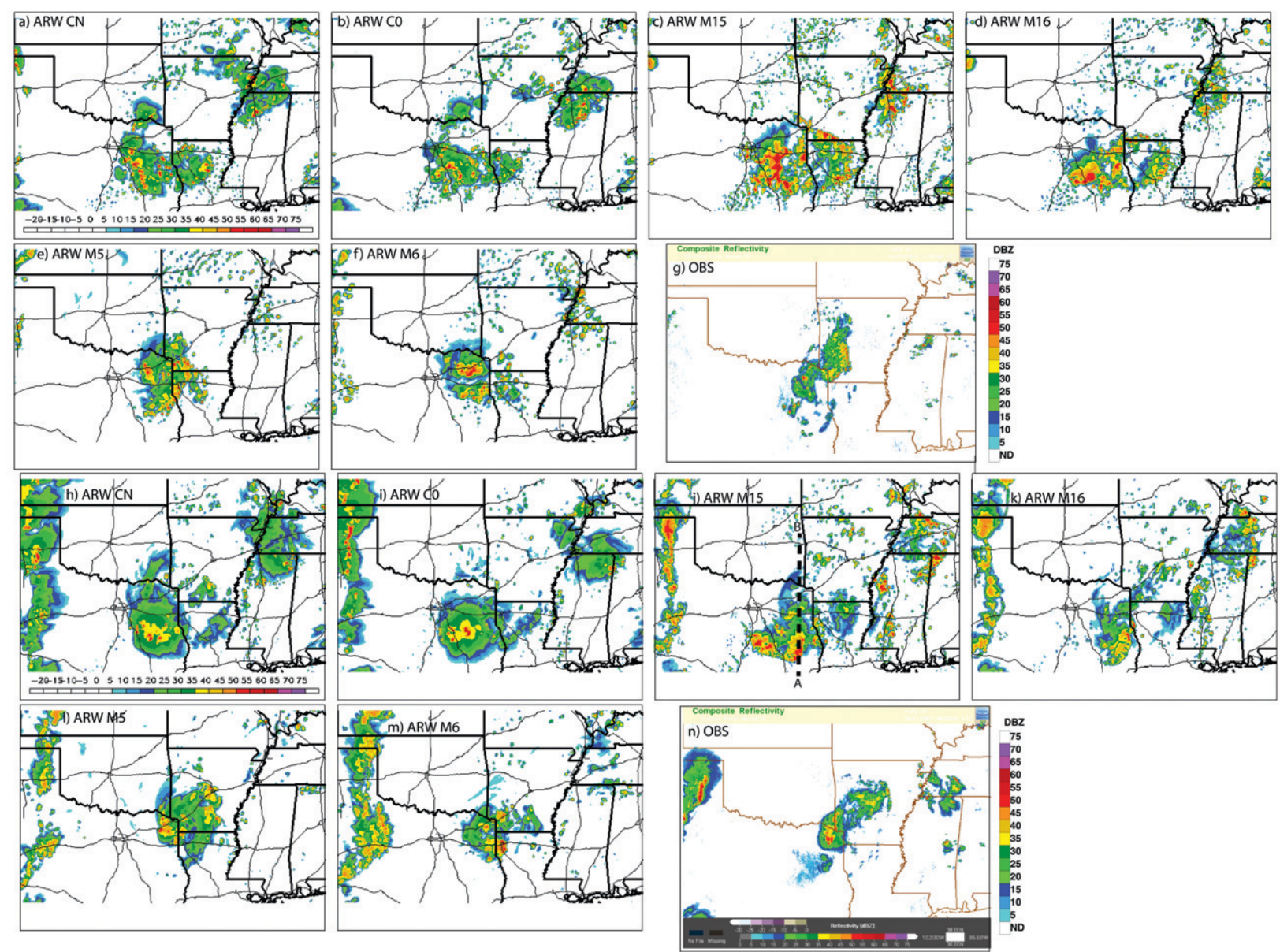

FIG. 11. As in Fig. 9, but (a)-(g) valid at 2100 UTC 10 Jun 2010 and (h)-(n) valid at 0000 UTC 11 Jun 2010. Line A-B in (j) shows the location of the vertical sections shown in Fig. 12.

Zhang and Fritsch 1987; Fritsch et al. 1994). In the ensemble members where the 10 June MCS dissipated too quickly, this cold dome was weak or nonexistent (Figs. $10 \mathrm{a}, \mathrm{b}, \mathrm{d})$, whereas in those where convection continued through the morning, the forecast cold dome was similar in location and magnitude to the observed cold dome (Figs. 10c,e,f).

As strong southerly winds approach the slow-moving $\mathrm{MCV}$, they will rise along the upward-sloped isentropes associated with this low-level cool anomaly and the midlevel PV anomaly (e.g., Raymond and Jiang 1990; Fritsch et al. 1994; Schumacher and Johnson 2009), and, therefore, this region can serve as a preferred location of the development of additional convection. Indeed, in the atmosphere and in most of the ensemble members, new deep convection initiated between 2100 UTC 10 June and 0000 UTC 11 June (Fig. 11). The organization and evolution of that convection varied widely, however. In members arw_c0 and arw_cn, which predicted the morning MCS to weaken and had a weak surface cold dome (Figs. 9a,b and 10a,b), the convection intensified, but only briefly. A south-north vertical section through the MCV in member arw_cn illustrates that despite the presence of unstable air in the boundary layer and isentropic upglide aloft, there was only a very weak dome of cold air near the surface to focus the ascent of the unstable air (Fig. 12a). The convection that developed between 2100 and 0000 UTC generally dissipated to only stratiform precipitation by 0300 UTC 11 June (Figs. 11a,b,h,i and 13a,b). In members arw_m15 and arw_m16, which partially maintained the morning MCS (Figs. 9c,d and 10c,d), deep convection initiated around 2100 UTC and quickly intensified and developed a strong evaporatively driven cold pool. The squall line predicted by these members was much more intense than the observed MCS, and it surged southward from 2100 UTC 10 June to 0000 UTC 11 June (Figs. 11c,d,j,k), stabilizing the atmosphere in the vicinity of the MCV 


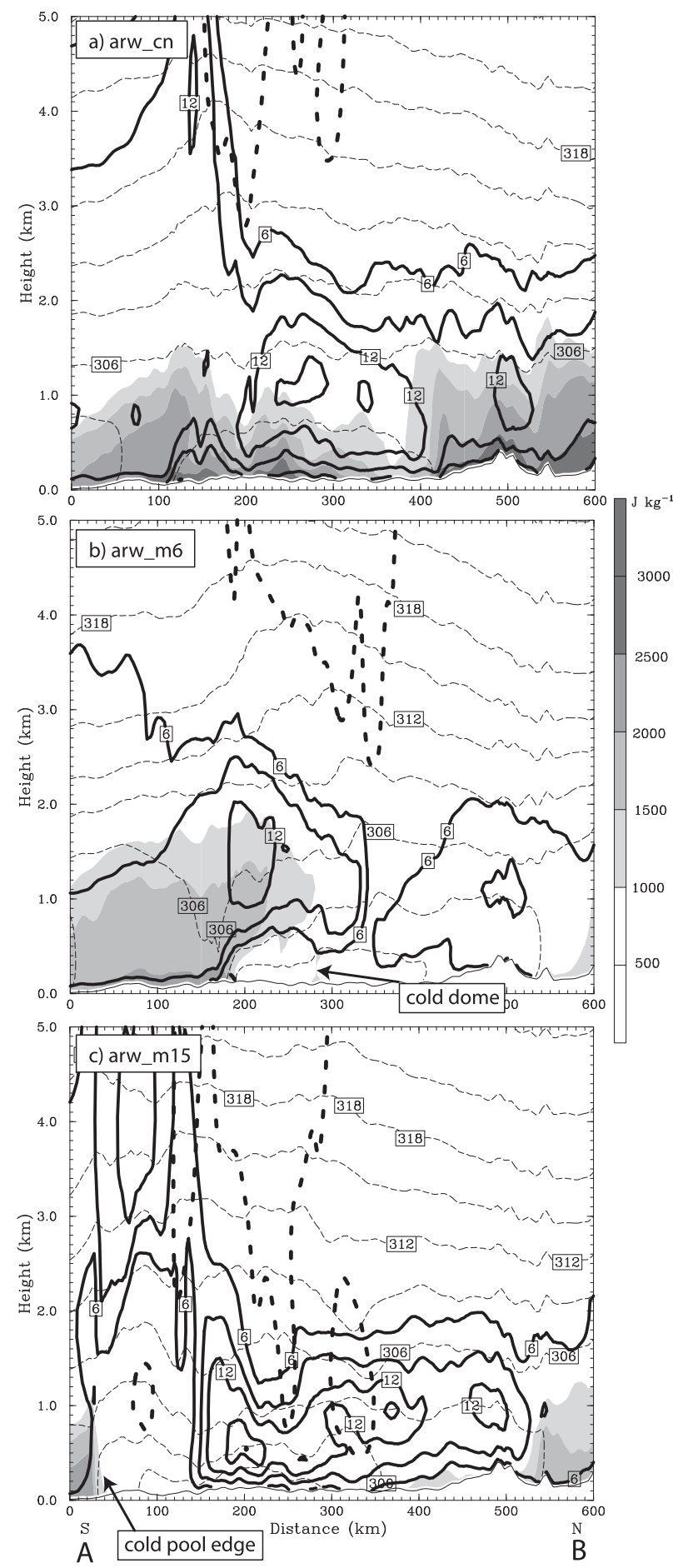

FIG. 12. South-north vertical section at 0000 UTC 11 Jun 2010 for members (a) arw_cn, (b) arw_m6, and (c) arw_m15. Shading shows CAPE ( $\mathrm{J} \mathrm{kg}^{-1}$ for parcels lifted from each level), thick solid contours show meridional wind speed (contoured every $3 \mathrm{~m} \mathrm{~s}^{-1}$ starting at 6), thin dashed contours show potential temperature every $2 \mathrm{~K}$, and the thick black contour shows the 1.5 potential vorticity unit (PVU) surface, where $1 \mathrm{PVU}=10^{-6} \mathrm{~m}^{2} \mathrm{~s}^{-1} \mathrm{~K} \mathrm{~kg}^{-1}$. The section is taken along line A-B shown in Fig. 11j, and values are averaged over 15 grid points on either side of this line.
(Fig. 12b). As a result there was no nocturnal MCS in these members (Figs. 13c,d). These two ensemble members used the WDM6 and WSM6 microphysics schemes (Table 1), respectively, and the strong cold pools and fast southward movement of the convection in this case are consistent with the southward displacements noted by Clark et al. (2012a) that were common throughout the 2010 HWT Spring Forecast Experiment in forecasts using these microphysics schemes. Finally, in the members that generally had the best representation of the morning MCS (arw_m5 and arw_m6), convection initiated and organized in a very similar manner to the observed convection (Figs. 11e,f,l,m), with a heavy-rainproducing MCS located over southwestern Arkansas by 0300 UTC 11 June (cf. Figs. 13e-g). In these members, there was a signal of organized ascent as the strong winds in and above the LLJ were lifted both within the general isentropic upglide and by the near-surface cold dome (member arw_m6 is shown in Fig. 12c). This ascent was also located near the center of the MCV, which supports further intensification of the vortex via latent heat release, similar to the conceptual model of Raymond and Jiang (1990) and Fritsch et al. (1994). Thus, from these results it appears that the strength of the cold dome left behind by the MCS on the morning of 10 June was key to whether or not strong, organized convection developed later on 10 June and early 11 June. Then, once convection initiated, microphysical processes also influenced the evolution of that convection.

The resulting 6-h precipitation forecasts from these members bear out the story told by the initiation and maintenance of the MCS, with members arw c0 and arw_cn producing a small area with between 25 and $50 \mathrm{~mm}$ of rain located to the south of the observed maximum (Figs. 13h,i), members arw_m15 and arw_m16 predicting very little precipitation during this 6 -h period (Figs. $13 \mathrm{j}, \mathrm{k})$, as the intense squall line predicted by these members had already moved southward toward the Gulf of Mexico, and members arw_m5 and arw_m6 predicting a heavy rainfall event that very closely resembled the observed event (cf. Figs. 131-n).

Thus far, the analysis has focused on a select subset of members to develop and test hypotheses about the key processes in the 10-11 June 2010 heavy-rain-producing MCS. In particular, differences in the evolution of the MCS on the morning of 10 June, which then influenced the characteristics of a near-surface dome of cool air, were shown to be important in the ultimate initiation, organization, and maintenance of the MCS that occurred on 11 June, at least in these ensemble members. The SSEF as a whole also supports this hypothesis, as the majority of members had a surface cold dome that was too weak at 1800 UTC 10 June, and also did not 

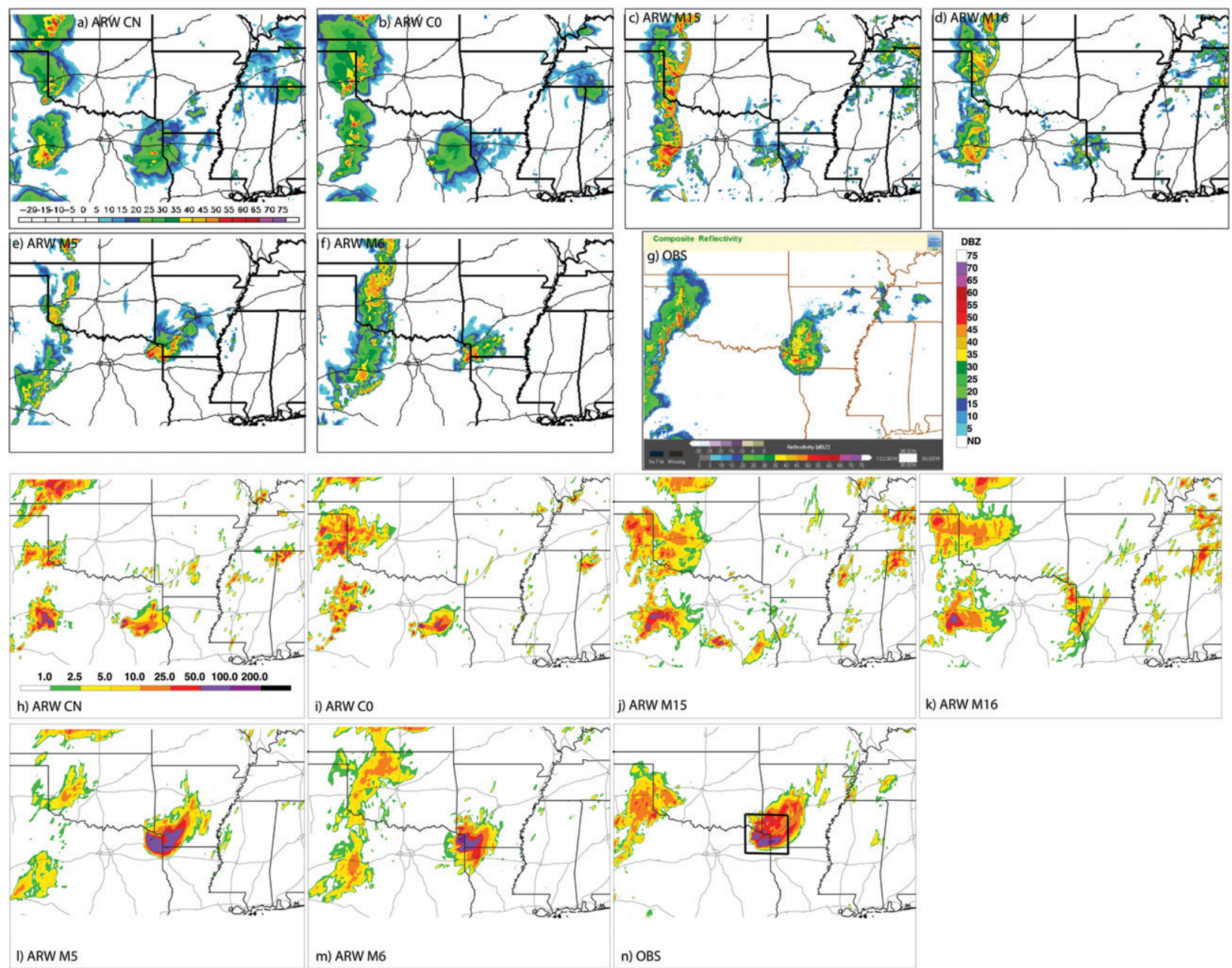

FIG. 13. (a)-(g) As in Fig. 9, but valid at 0300 UTC 11 Jun 2010. (h)-(m) SSEF 24-30-h precipitation forecasts (mm) initialized at 0000 UTC 10 Jun 2010 and valid for the 6-h period ending at 0600 UTC 11 Jun 2010. (n) Stage IV precipitation analysis (mm) for the 6-h period ending at 0600 UTC 11 Jun 2010. The rectangle in (n) indicates the region used for the area averaging of precipitation in Fig. 14.

predict the heavy rainfall that occurred from 0000 to 0600 UTC 11 June (clustered in the bottom-right corner of Fig. 14). A few ensemble members, however, that had a cold dome at 1800 UTC that was closer in magnitude to the observations, were able to correctly predict the heavy rain event (toward the top left of Fig. 14). Only four ensemble members had an area-averaged potential temperature perturbation (defined as the difference between the area-averaged potential temperature in the inner and outer boxes shown in Fig. 10a) of less than $-3 \mathrm{~K}$, and three of these predicted large amounts of precipitation over the area where it was observed. Member arw_10, which had a strong cold dome at 1800 UTC, also predicted an MCS of similar structure, timing, and intensity to the observed MCS, but it was displaced to the south such that it fell outside the region over which precipitation was averaged for Fig. 14 (not shown). These four members had cold domes that were significantly different from that in the ensemble mean (Fig. 14). And member nmm_m4, which had a relatively weak cold dome but heavy precipitation, needs to be considered in the context that over the course of the 2010 HWT Spring Forecast Experiment, all of the members using the NMM dynamical core predicted heavy precipitation much more often than it was actually observed (Fig. 6), and also much more often than the ARW members did.

The members with a stronger surface cold dome also tended to have a more intense MCV both in the model initial conditions (Fig. 7) and on the afternoon of 10 June (Fig. 15). Physically, this is consistent with there being a longer-lasting MCS on the morning of 10 June, which 


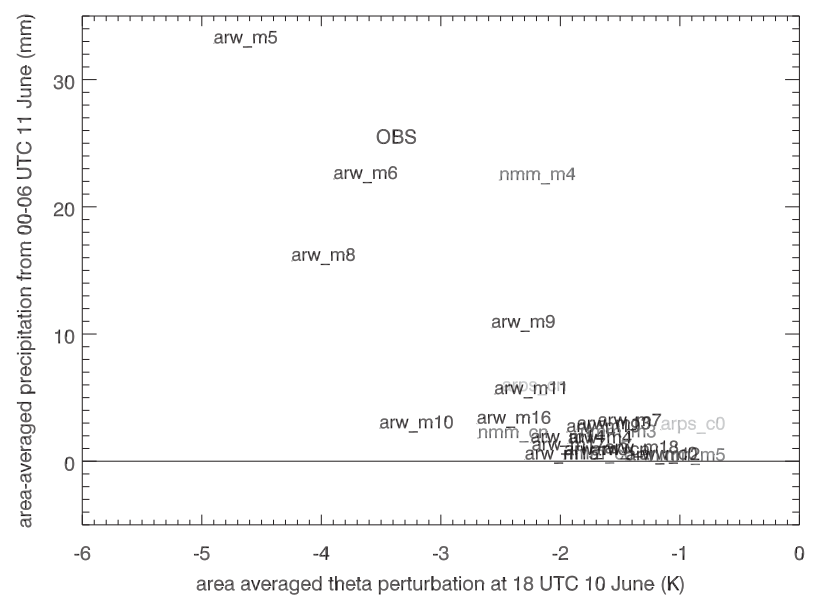

FIG. 14. Scatterplot comparing area-averaged potential temperature perturbation at 1800 UTC 10 Jun to area-averaged precipitation (mm) between 0000 and 0600 UTC 11 Jun 2010 for each SSEF member. The area-averaged potential temperature perturbation was calculated by subtracting the potential temperature averaged over the inner region shown in Fig. 10a from that in the outer region. The area-averaged precipitation was calculated over the region shown in Fig. 13n. Members using ARW are shown in black, those using the NMM in dark gray, and those using ARPS in light gray. The observed value is also shown; this was calculated by areally averaging the stage IV precipitation and objectively analyzed surface observations over the same areas used for the forecasts. A 5000permutation bootstrap test indicates that, with $99 \%$ confidence, the ensemble mean $\theta$ perturbation is between -2.8 and $-1.9 \mathrm{~K}$ and the ensemble mean precipitation is less than $10.4 \mathrm{~mm}$; members with values greater or less than these values are significantly different from the ensemble mean.

led to both an intensification of the MCV via latent heat release and cooler air at low levels owing to more evaporation and greater cloudiness.

Whereas the relationship between the afternoon character of the MCV's features and the precipitation the next night appears to be strong, there is essentially no correlation between the available atmospheric moisture and precipitation (Fig. 16). This is because all of the ensemble members predicted a moist environment in northern Texas and western Arkansas, with areaaveraged PW between 50 and $55 \mathrm{~mm}$, yet there was still large variability in the precipitation forecast. Similarly, all members had more than $2000 \mathrm{~J} \mathrm{~kg}^{-1}$ of mostunstable CAPE (MUCAPE) in the environment to the south of the MCV (not shown). Therefore, considering the ensemble-based analysis in terms of the ingredientsbased methodology for forecasting and understanding deep convection and heavy rainfall (e.g., Johns and Doswell 1992; Doswell et al. 1996), the necessary moisture and instability were present in all of the SSEF members, but there was less certainty in whether the necessary lift would also be present.

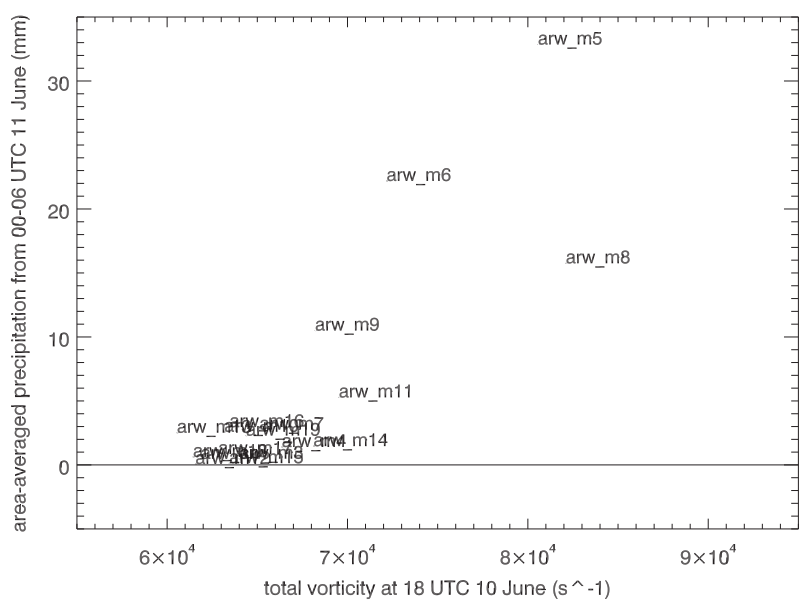

FIG. 15. As in Fig. 14, but the abscissa shows area-integrated absolute vorticity $\left(\mathrm{s}^{-1}\right)$ in the $700-500-\mathrm{hPa}$ layer. The integration was conducted over the same $183 \mathrm{~km} \times 183 \mathrm{~km}$ box used to identify the MCV locations in Fig. 8. Because of limitations in obtaining, processing and analyzing three-dimensional model output, only the SSEF members using the ARW dynamical core are shown here. Similarly, because an analysis dataset that included explicit representation of convection is not available, the observed vorticity value is not estimated here. Members with integrated vorticity greater than $7.03 \times 10^{4} \mathrm{~s}^{-1}$ are significantly different from the ensemble mean with $99 \%$ confidence.

Based on the objective and subjective evaluation of the SSEF members, a summary of the pathways by which the SSEF members evolved is presented in Fig. 17. The maintenance of the MCS on the morning of 10 June in northern Texas is used as a starting point for this analysis, and then the resultant evolution of the MCV, the surface boundary, and afternoon convection determined whether or not an accurate prediction of the heavy-rain-producing MCS early on 11 June was made. These results also offer insights into the processes in the real atmosphere that are most important for the development of heavy precipitation near an MCV-although the differences between many of these ensemble members are quite subtle, it appears that the persistence of some precipitation through the day, along with a coherent MCV and associated surface dome of cool air, are key to the initiation and maintenance of a heavy-rain-producing MCS the following night. This result is somewhat paradoxical in the context of the results of Davis and Trier (2002) and Schumacher and Johnson (2008), who found that a cold pool at the surface may not be necessary for the maintenance of the heavy-rain-producing MCS itself. The main finding, then, may be that a remnant pocket of cool air at the surface helps to focus the initial convection that develops near the MCV, but then the presence of that low-level cool air becomes of secondary importance 


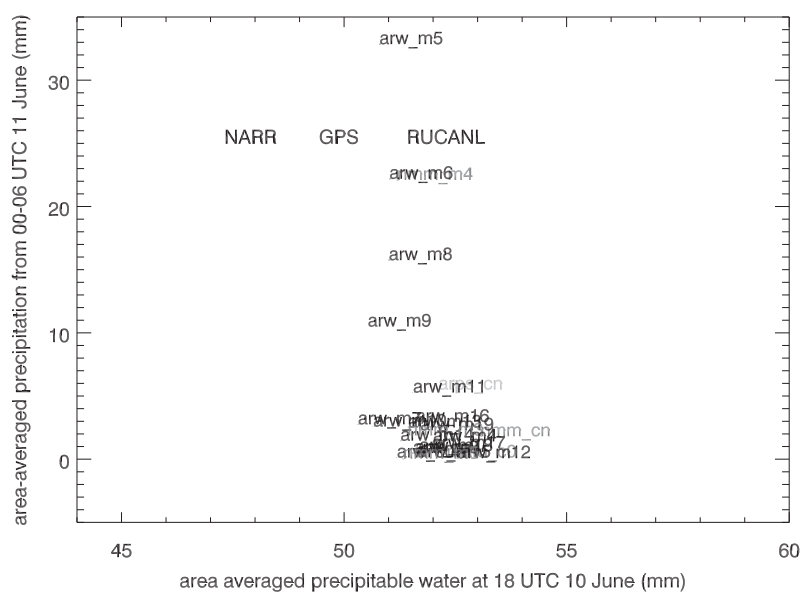

FIG. 16. As in Fig. 14, but the abscissa shows area-averaged PW $(\mathrm{mm})$ over the region covering $29.5^{\circ}-35.5^{\circ} \mathrm{N}$ and $93^{\circ}-98.5^{\circ} \mathrm{W}$ (most of northeast TX, and parts of southwest AR, northeast LA, and southeast $\mathrm{OK})$. The area-averaged PW from the NARR and RUC analyses, and an average of 12 GPS PW observations in this region, are also plotted against the area-averaged stage IV precipitation. Multiple observation-based estimates are shown because of the discrepancies between them. GPS PW observations were obtained from the University Corporation for Atmospheric Research/Constellation Observing System for Meteorology, Ionosphere, and Climate (UCAR/COSMIC) program. Members with area-averaged PW less than 51.1 or greater than $51.8 \mathrm{~mm}$ are significantly different from the ensemble mean with $99 \%$ confidence.

as the ascent created by the MCV-LLJ interaction is enhanced and the MCS develops and evolves.

Furthermore, these results put another constraint on the findings of Trier et al. (2000a) that midlevel moisture is a key discriminator between MCVs that go on to initiate convection on a second or third diurnal cycle. Trier et al. (2000a) showed that MCVs surrounded by dry air at midlevels were unlikely to have secondary convection, and our results show that even with a very moist environment, the initiation and organization of secondary convection is not guaranteed. These ideas should be examined further in future research of additional cases.

\section{c. Structure of the low-level jet}

Another aspect of the SSEF on 10-11 June 2010 where there were systematic differences among the ensemble members was in the vertical structure of the LLJ. In particular, the members with different PBL parameterizations represented the LLJ differently in the inflow region to the heavy-rain-producing MCS in eastern Texas. The members using the YSU PBL scheme all had an LLJ that had a weaker maximum wind, and a wind maximum at a greater height, than the members using other schemes (Fig. 18a). The YSU members had maximum LLJ winds of $12-17 \mathrm{~m} \mathrm{~s}^{-1}$ at approximately $1000 \mathrm{~m} \mathrm{MSL}$, whereas the other members had maximum winds between 18 and $21 \mathrm{~m} \mathrm{~s}^{-1}$ at $600-800 \mathrm{~m} \mathrm{MSL}$.

In this case, the differences in the nocturnal LLJ structure in the members with different PBL schemes appear to be a result of differences in the depth of the PBL during the afternoon. Afternoon soundings from representative members using the YSU and MYJ schemes reveal that the PBL is much moister and shallower in the MYJ members than in the YSU members (Fig. 18b). Since the nocturnal LLJ tends to form near the top of where the daytime boundary layer was (e.g., Stull 1988), the deeper PBL in the YSU members is consistent with higher height of their nocturnal LLJ maximum. It does not necessarily explain the reasons for the differences in LLJ velocity, however.

The results found here are generally similar to those of $\mathrm{Hu}$ et al. (2010), who found that the MYJ scheme had a cool and moist bias in simulations of the boundary layer in southeast Texas, whereas the YSU scheme had smaller biases in temperature and moisture. They also showed that the YSU scheme had nocturnal wind maxima that had a higher height of maximum wind, which was more consistent with observations, than did the MYJ scheme. However, the maximum velocity in the MYJ scheme more closely matched observations (Hu et al. 2010, see their Fig. 10). Storm et al. (2009) also found that WRF-predicted LLJs were sometimes too weak and had their altitude of maximum winds located too high.

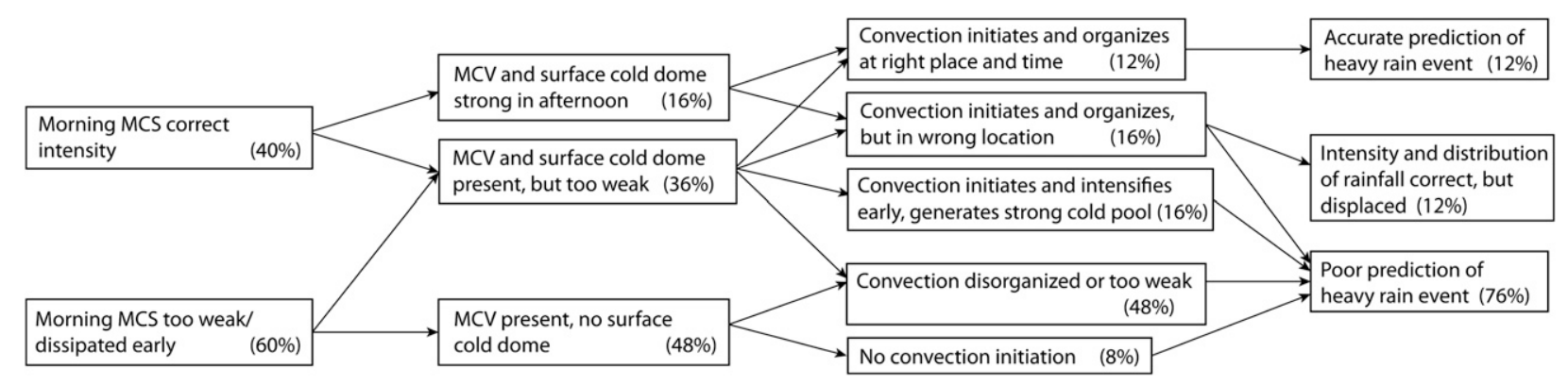

FIG. 17. Diagram summarizing the pathways by which the SSEF members initialized at 0000 UTC 10 Jun 2010 evolved, based on objective and subjective evaluation of the ensemble forecasts. Percentages of members in each category are given. 

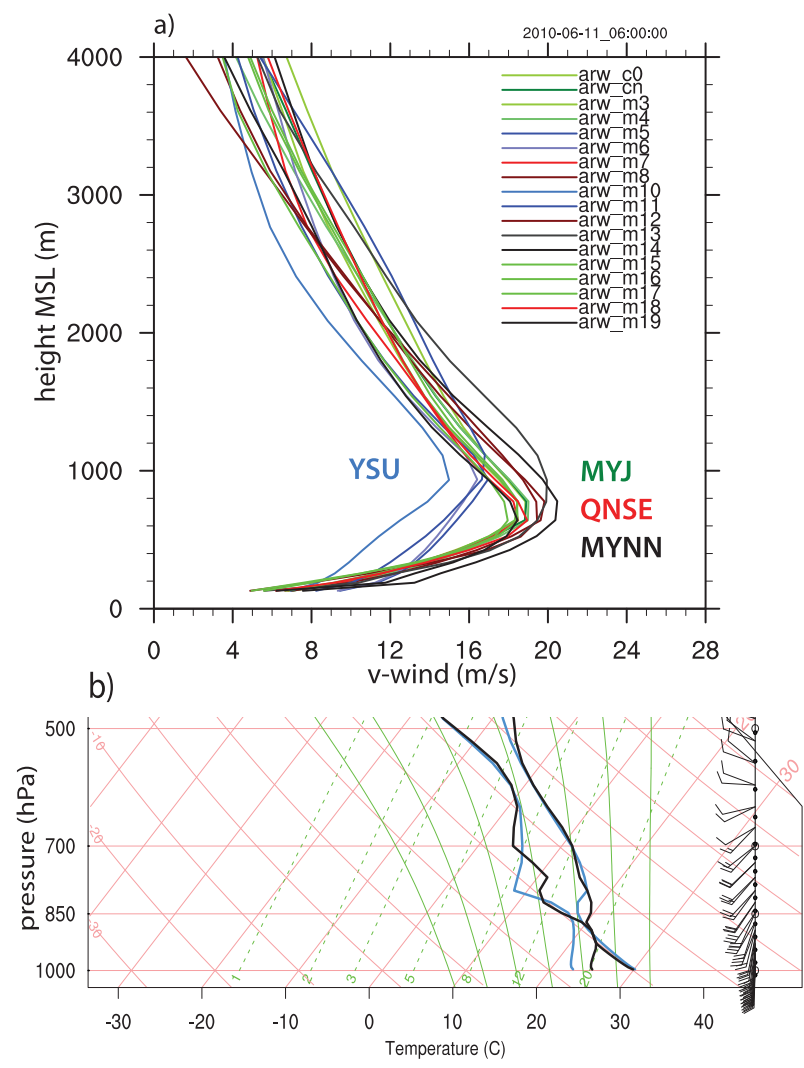

FIG. 18. (a) Meridional wind profile, averaged over a portion of east TX shown in Fig. 2e, for the 30-h SSEF forecast initialized at 0000 UTC 10 Jun and valid at 0600 UTC 11 Jun 2010. Members using the YSU PBL parameterization are in shades of blue, those using the MYJ scheme are in shades of green, those using the Mellor-Yamada-Nakanishi-Niino (MYNN; Nakanishi and Niino 2006) scheme are in shades of black/gray, and those using the quasinormal scale elimination (QNSE; Sukoriansky et al. 2006) scheme are in shades of red. (b) Skew $T-\log p$ diagram at College Station, TX (shown in Fig. 2), valid at 1800 UTC 10 Jun, from member arw_m6 (which uses the YSU PBL scheme and is shown in blue) and member arw_m15 (MYJ PBL scheme, shown in black).

Unfortunately, no suitable observations of the LLJ structure are available in eastern Texas, as operational wind profilers do not adequately sample below $500 \mathrm{~m}$ AGL (e.g., Whiteman et al. 1997), so evaluating which wind profiles best represented observations is not possible for this case. Furthermore, it is not clear what, if any, influence the differences in LLJ structure had on the development and maintenance of the heavy-rain-producing MCS, but one possible mechanism is discussed in section 4d. Further evaluation of model forecasts of LLJs, along with the collection of suitable observations in datasparse regions, is strongly encouraged for future research.

\section{d. Sensitivity experiments}

Based on the results presented in the previous section, several sensitivity experiments were also conducted to
TABLE 2. Configuration of the sensitivity experiments. Entries are only given where the configuration differs from the original configuration of the member. Columns and entries are named as in Table 1.

\begin{tabular}{lcccc}
\hline \hline \multicolumn{1}{c}{ Name } & LBCs & Microphysics & $\begin{array}{c}\text { Land } \\
\text { surface }\end{array}$ & PBL \\
\hline Arw_cn_ysu & - & - & - & YSU \\
Arw_cn_ysu_ruc & - & - & RUC & YSU \\
Arw_m6_myj & - & - & - & MYJ \\
Arw_m6_myj_noah & - & - & Noah & MYJ \\
Arw_m6_thomps & - & Thompson & - & - \\
Arw_m6_wdm6 & - & WDM6 & - & - \\
Arw_m6_cnbdy & Control & - & - & - \\
\hline
\end{tabular}

further isolate the differences in the representation of key processes by different model configurations. These experiments focus on two members of the original ensemble: the control ARW member (arw_cn), and member arw_m6, which was found to best reflect the observations. These sensitivity experiments are designed to address several questions raised by the analysis of the SSEF output, particularly whether the ICs or the choice of physical parameterizations were most closely tied to the quality of the forecast of this case. Seven sensitivity experiments were run using variations from either member arw_cn or arw_m6 (Table 2).

The first four experiments were conducted because the members that generally performed the best for the 11 June 2010 MCS used a combination of the RUC land surface model and the YSU PBL parameterization. The control member and many other SSEF members, on the other hand, used the Noah land surface model and the MYJ PBL parameterization. None of the original members used a combination of the RUC land surface model and the MYJ PBL scheme, and only one used the Noah land surface with the YSU PBL. Thus, we explore the sensitivities to these different parameterizations by altering the control member to use the land surface and PBL schemes that were used in the successful members, and by altering one of the successful members to use the land surface and PBL schemes of the control member. The sensitivity to changing only one of these parameterizations is also tested.

The sensitivity forecasts using the ICs and LBCs from the control member but different land surface and PBL schemes performed similarly to the control member itself (Figs. 19a-c). They also had a weak afternoon cold dome (Fig. 20a) and a relatively weak MCV (Fig. 20b), and did not produce heavy precipitation in correct location in the 24-30-h forecast. However, the change from the control configuration (using the MYJ PBL and Noah land surface schemes) to the YSU PBL and RUC land surface schemes did result in an MCS that produced 

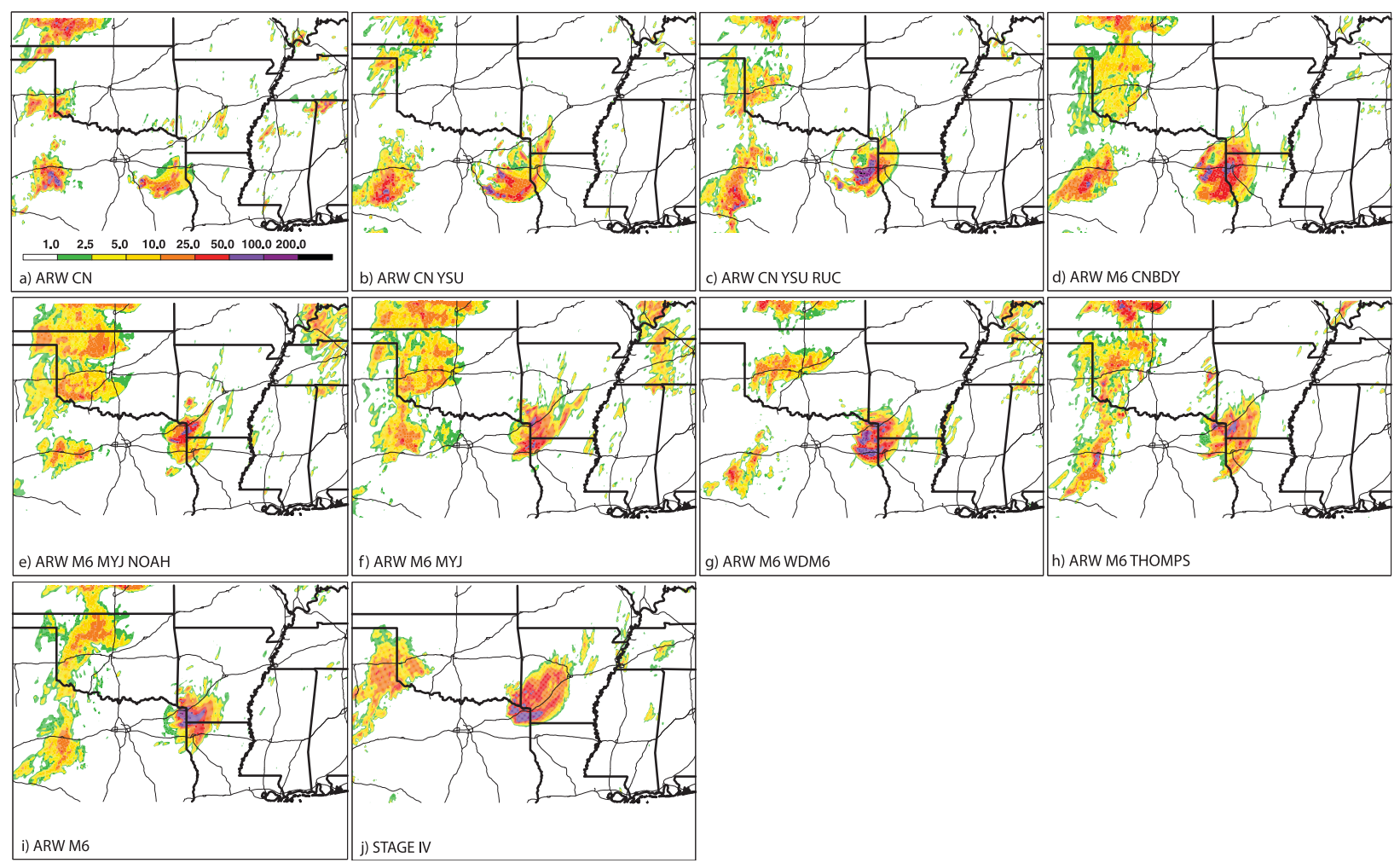

FIG. 19. (a)-(i) SSEF 24-30-h precipitation forecasts (mm) initialized at 0000 UTC 10 Jun 2010 and valid for the 6-h period ending 0600 UTC 11 Jun 2010, for experiments (a) arw_cn, (b) arw_cn_ysu, (c) arw_cn_ysu_ruc, (d) arw_m6_cnbdy, (e) arw_m6_myj_noah, (f) arw_m6_myj, (g) arw_m6_wdm6, (h) arw_m6_thomps, and (i) arw_m6. (j) Stage IV precipitation analysis (mm) for the 6-h period ending 0600 UTC 11 Jun 2010.

heavier precipitation, albeit displaced to the south of the observed MCS (Fig. 19c).

An intermediate experiment (arw_m6_cnbdy) was conducted that used the ICs and the original configuration from member arw_m6, but the LBCs from the control member. This was designed to test the influence of the lateral boundary conditions on the forecast at hours 24-30. The output from this experiment showed some characteristics of the member from which it took its ICs-it predicted a strong MCV and cold dome in the afternoon (Figs. 20a,b) and it also predicted a heavyrain-producing nocturnal MCS, but it was displaced approximately $150 \mathrm{~km}$ to the south of the observed location (Fig. 19d). Thus, in addition to sensitivities to the initial conditions, perturbations at the lateral boundaries also exhibit an influence on the precipitation forecast. Using clustering analysis, Johnson et al. (2011) also found important influence of the LBCs on the precipitation forecasts of the second day in CAPS SSEF output.

A set of sensitivity experiments using the ICs and LBCs from member arw_m6, which produced the best forecast of the 11 June MCS, also had characteristics similar to the original member arw_m6. The variations on that member produced less total rainfall than the original member in the region of interest, but they did correctly predict an MCS in approximately the right place and time (Figs. 19d-i and 20). They also predicted relatively strong afternoon MCVs and cold domes (Figs. 20a,b). Furthermore, replacing the YSU PBL scheme and the RUC land surface model with the MYJ and Noah schemes reduced the intensity of the precipitation in the MCS, consistent with the first set of sensitivity experiments. One possible reason for these differences will be discussed below.

Two experiments were also conducted in which the configuration of the arw_m6 member was held constant except for the cloud microphysics parameterization. The Thompson scheme (used in the control member) and the WDM6 scheme (which was shown in the previous section to produce very intense cold pools) were examined. As with the original member, nocturnal MCSs were predicted in approximately the right place and time by these modified members (Figs. 19g,h), but they produced somewhat less total precipitation than the original member in the region of interest (Figs. 20a,b). The test using the WDM6 parameterization did produce a stronger cold pool both in the afternoon 

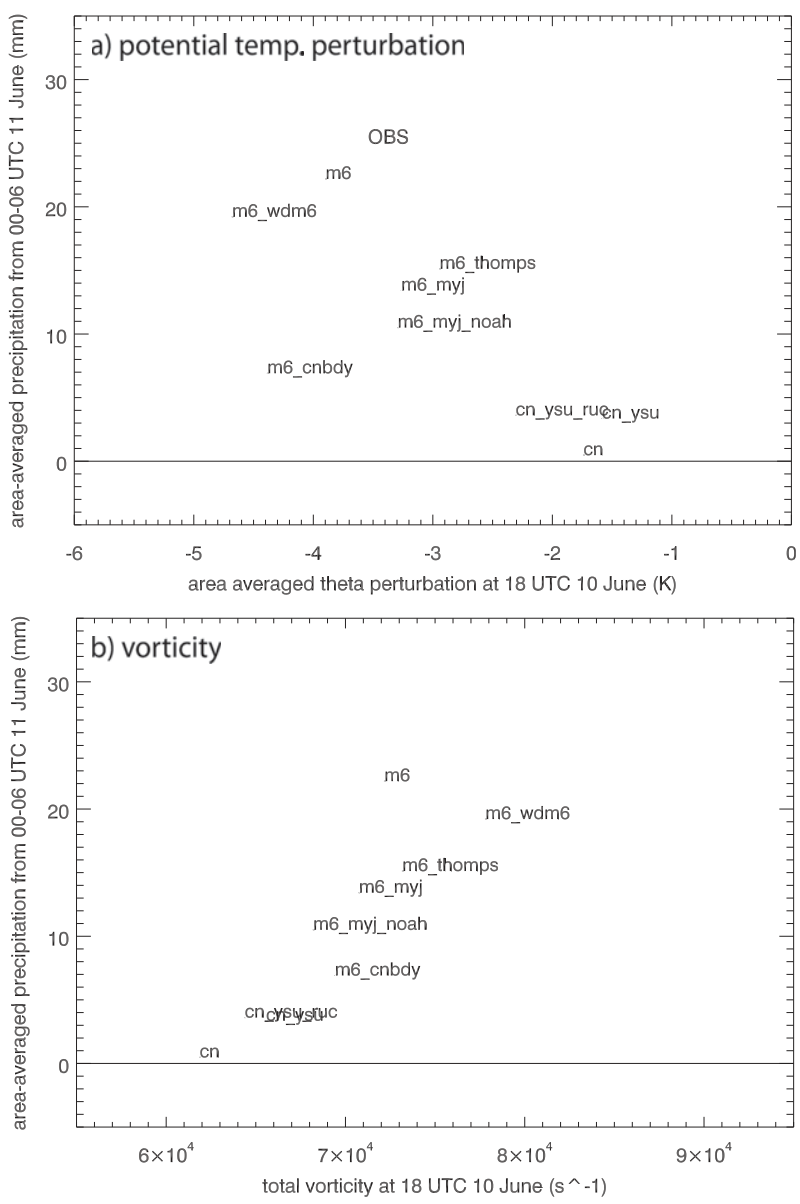

FIG. 20. (a) As in Fig. 14, but showing only members arw_cn, arw_m6, and the associated sensitivity experiments outlined in Table 2. (b) As in Fig. 15, but showing only members arw_cn, arw_m6, and the associated sensitivity experiments outlined in Table 2. The "arw" prefixes have been removed for clarity.

(Fig. 20a) and from the subsequent nocturnal MCS (not shown) than the original configuration, consistent with the other members using the WDM6 scheme (section 4b).

The low-level wind profiles for these sensitivity experiments were also analyzed to determine whether the location and intensity of the predicted LLJs changed along with the changes in model configuration. The results (not shown) were consistent with those stated previously; namely, that members using the YSU PBL parameterization had LLJs that were weaker and were maximized at a higher altitude than the members using the MYJ scheme. One possible connection between the PBL parameterization, the LLJ, and the resulting precipitation amounts is suggested by comparing Figs. $12 \mathrm{~b}$ and 21. As discussed earlier, member arw_m6 (Fig. 12b), which used the YSU PBL parameterization, had a deep, relatively dry boundary layer, and had a very accurate precipitation forecast. Sensitivity experiment arw_m6_ myj_noah (Fig. 21) used the same ICs and LBCs as that member, but the MYJ PBL scheme and the Noah land surface model (Table 2). It reflected a shallower, moister boundary layer along with an LLJ that was stronger and peaked at a lower altitude. However, even though the air in the PBL in member arw_m6 was drier and less unstable than in the sensitivity experiment, there was a deeper layer of high-CAPE air that could be processed by the convective system. This could be a reason that the arw_m6 member resulted in a forecast with more total precipitation than member arw_m6_myj_noah. However, this hypothesis has not been tested thoroughly and should be examined in additional cases to verify its validity.

In summary, the sensitivity experiments show that for this case of heavy convective precipitation associated with an MCV, the initial and lateral boundary conditions were the first-order factors determining the location and evolution of convection. The choice of physical parameterizations also influenced the precipitation forecast, but to a secondary degree. These results are consistent with the ideas presented in section $4 \mathrm{c}$ regarding the representation of convection early in the forecast (which was likely closely tied to the initial conditions) having important effects on the environment that then determine whether convection would develop the next night. However, it is not known whether these results would hold for other MCV cases (or other ensemble configurations), and this is a subject suggested for future research. Since the development and maintenance of MCVs is also closely tied to latent heating/cooling processes that are in turn closely tied to the parameterization of cloud microphysics (e.g., Hopper and Schumacher 2012), it must also be important to include uncertainties in those processes in a storm-scale ensemble. Different parameterizations interact with one another in different ways (e.g., Jankov et al. 2005), and further research into the nature of these interactions is encouraged.

\section{Conclusions and suggestions for future work}

In this study, an ensemble of convection-allowing numerical weather forecasts was analyzed to determine the processes that were favorable for, or detrimental to, the development of heavy-rain-producing MCSs on 911 June 2010. First, a general overview of the exceptionally long-lived MCV was presented, that showed its origins in west Texas nearly a week before this heavy rainfall occurred. Next, an evaluation of the highresolution ensemble for this 3-day period of heavy rainfall was conducted, revealing high forecast uncertainty. Then, the 10-11 June period was analyzed in more detail. The ensemble members were contrasted, and it was found that the representation of a dissipating MCS near 


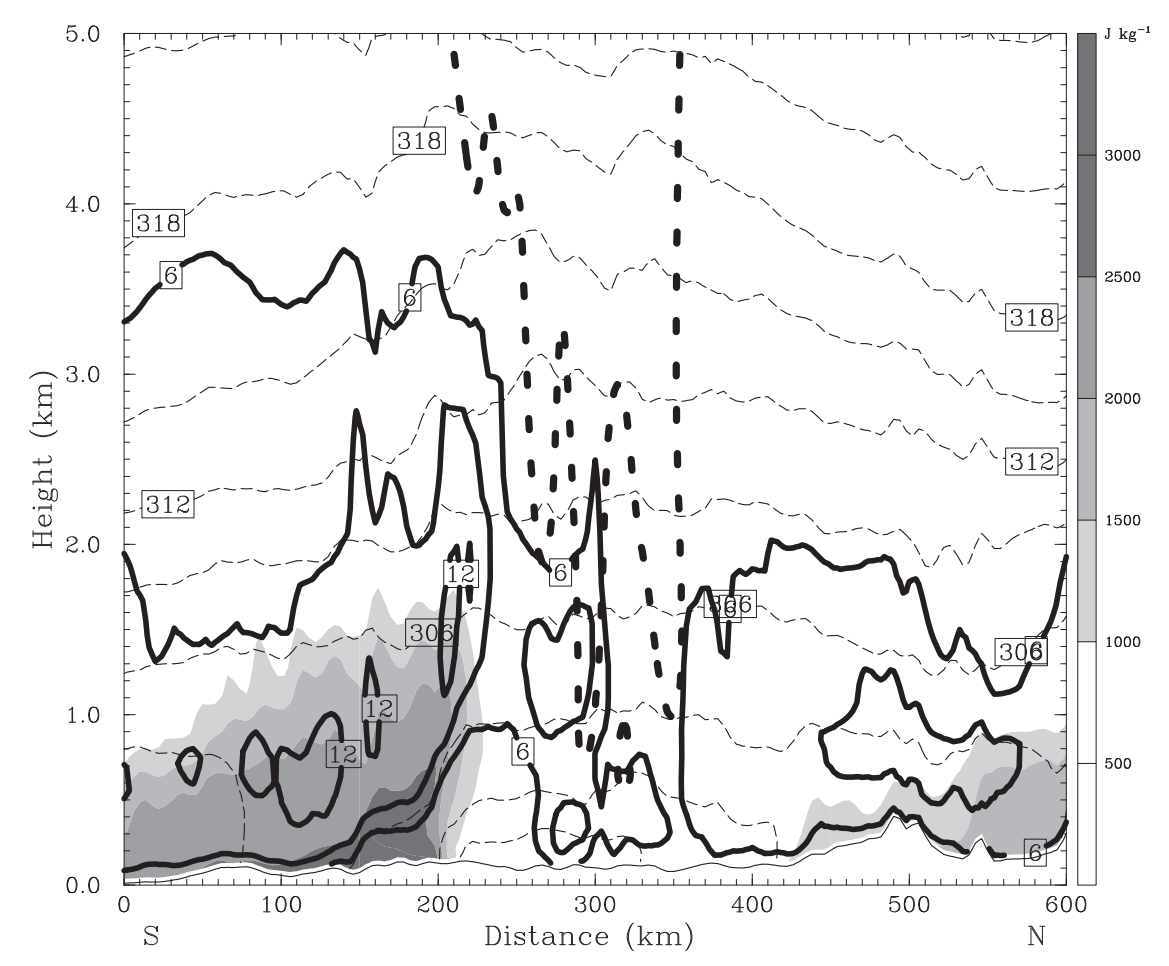

FIG. 21. As in Fig. 12, but for sensitivity experiment arw_m6_myj_noah.

the beginning of the forecast was a key factor in determining whether an MCS initiated and was maintained the following night. In particular, members that correctly maintained the morning MCS also had an accurate representation of the intensity of the associated $\mathrm{MCV}$, as well as a dome of cool air near the surface, whereas members in which the morning MCS dissipated too early did not correctly predict these features. The cold dome, along with ascent associated with the MCV, helped to initiate and organize deep convection the following afternoon and evening. Then, a correct forecast of evaporatively driven cold pools (specifically, a correct forecast that they remain relatively weak) was required for the convection to evolve into a nocturnal heavy-rain-producing MCS. The representation of the nocturnal LLJ among ensemble members with different parameterizations of the PBL was shown to be substantially different, with members using the YSU scheme having a weaker LLJ and a higher altitude of maximum winds than members using other schemes. Finally, a series of sensitivity experiments showed that the representation of the initial and lateral boundary conditions was the factor most responsible for the quality of the precipitation forecast in this case, although the choices of physical parameterizations also had a nonnegligible effect.

This research contributes to the growing literature on the evaluation and analysis of high-resolution ensemble forecasts, and also provides insights into the atmospheric processes that are important in the development and maintenance of extreme-rainfall-producing convective systems. It also raises a number of additional questions for future investigation. We have only presented one detailed case study here, and it is not known whether these results would be representative of other cases. Although the circumstances surrounding this particular event were unique, a combination of additional case studies and more idealized numerical simulations may reveal further information about the factors governing long-lived MCVs and MCSs. Further investigation of factors such as soil moisture, boundary layer processes, and the low-level jet in this and other cases is also warranted. The results also illustrate the importance of accurately representing convection and its effects in the first few hours of a forecast, which is the subject of much current research in the assimilation of radar and other mesoscale observations. The analysis in this manuscript highlights both the insights that can be obtained from careful analysis of convection-allowing, mixed-physics ensemble prediction systems, but also some of the challenges of interpreting such forecasts because of their high resolution and representation of nonlinear processes. Finally, given that ensemble forecasts with explicit representation of deep convection are nearing feasibility in the operational environment, studies of how 
forecasters can use ensemble-based analysis in real time, of how to communicate the high spatial variability and inherent uncertainty in forecasts of heavy rainfall, and in how users will understand and interpret these forecasts, are enabled by projects such as the HWT Spring Forecast Experiment and are of great importance for the near future.

Acknowledgments. The authors thank the NSSL NMQ for providing radar reflectivity mosaic images, to NCAR for providing archived stage IV precipitation data, and to UCAR/COSMIC for providing the GPS PW observations. The authors also thank Drs. Michael Coniglio (NSSL) and David Novak (HPC) for helpful discussions regarding this work. The constructive reviews from Dr. Thomas Galarneau and an anonymous reviewer, and editorial advice from Dr. Ron McTaggart-Cowan, helped to improve the manuscript considerably. CAPS SSEF forecasts were supported by the NOAA CSTAR Program with supplementary support from NSF Grant AGS0802888. The forecasts were produced on a Cray XT4 at the National Institute for Computational Science operated under the support of the National Science Foundation. R. Schumacher was supported by National Science Foundation Grants AGS-0954908 and AGS-1157425. M. Xue was supported by NSF Grants OCI-0905040, AGS-0941491, AGS-1046171, and AGS-1046081. Kevin Thomas, Yunheng Wang, Keith Brewster, Jidong Gao, and other scientists at CAPS contributed to the production of the 2010 CAPS SSEF forecasts. Finally, the authors express their gratitude to all of those responsible for organizing, running, and participating in the HWT Spring Forecast Experiment.

\section{REFERENCES}

Ancell, B., and G. J. Hakim, 2007: Comparing adjoint- and ensemblesensitivity analysis with applications to observation targeting. Mon. Wea. Rev., 135, 4117-4134.

Bosart, L. F., and F. Sanders, 1981: The Johnstown flood of July 1977: A long-lived convective system. J. Atmos. Sci., 38, 16161642.

Buizza, R., P. L. Houtekamer, G. Pellerin, Z. Toth, Y. Zhu, and M. Wei, 2005: A comparison of the ECMWF, MSC, and NCEP global ensemble prediction systems. Mon. Wea. Rev., 133, 1076-1097.

Chen, F., and J. Dudhia, 2001: Coupling an advanced land surfacehydrology model with the Penn State-NCAR MM5 modeling system. Part I: Model implementation and sensitivity. Mon. Wea. Rev., 129, 569-585.

Clark, A. J., W. A. Gallus Jr., M. Xue, and F. Kong, 2009: A comparison of precipitation forecast skill between small convectionallowing and large convection-parameterizing ensembles. Wea. Forecasting, 24, 1121-1140.

$[,-$ _ ification of precipitation forecasts from convection-allowing
NCAR WRF model simulations and the operational NAM. Wea. Forecasting, 25, 1495-1509.

_ - _ M. Xue, and F. Kong, 2010b: Convection-allowing and convection-parameterizing ensemble forecasts of a mesoscale convective vortex and associated severe weather environment. Wea. Forecasting, 25, 1052-1081.

_- and Coauthors, 2011: Probabilistic precipitation forecast skill as a function of ensemble size and spatial scale in a convectionallowing ensemble. Mon. Wea. Rev., 139, 1410-1418.

_ , and Coauthors, 2012a: An overview of the 2010 Hazardous Weather Testbed Experimental Forecast Program spring experiment. Bull. Amer. Meteor. Soc., 93, 55-74.

_ , and Coauthors, 2012b: Supplement to "An overview of the 2010 Hazardous Weather Testbed Experimental Forecast Program spring experiment." Bull. Amer. Meteor. Soc., 93 (Suppl.), ES1-ES5.

Coniglio, M. C., K. L. Elmore, J. S. Kain, S. J. Weiss, M. Xue, and M. L. Weisman, 2010: Evaluation of WRF model output for severe weather forecasting from the 2008 NOAA Hazardous Weather Testbed spring experiment. Wea. Forecasting, 25, 408-427.

Dabberdt, W. F., and Coauthors, 2005: Multifunctional mesoscale observing networks. Bull. Amer. Meteor. Soc., 86, 961-982.

Davis, C. A., and S. B. Trier, 2002: Cloud-resolving simulations of mesoscale vortex intensification and its effect on a serial mesoscale convective system. Mon. Wea. Rev., 130, 2839-2858.

Doswell, C. A., III, H. E. Brooks, and R. A. Maddox, 1996: Flash flood forecasting: An ingredients-based methodology. Wea. Forecasting, 11, 560-581.

Du, J., S. L. Mullen, and F. Sanders, 1997: Short-range ensemble forecasting of quantitative precipitation. Mon. Wea. Rev., 125, 2427-2459.

, J. McQueen, G. DiMego, Z. Toth, D. Jovic, B. Zhou, and H.-Y. Chuang, 2006: New dimension of NCEP Short-Range Ensemble Forecasting (SREF) system: Inclusion of WRF members. Preprints, WMO Expert Team Meeting on Ensemble Prediction System, Exeter, United Kingdom, WMO, 1-5. [Available online at http://www.emc.ncep.noaa.gov/mmb/SREF/ WMO06_full.pdf.]

Ebert, E. E., 2001: Ability of a poor man's ensemble to predict the probability and distribution of precipitation. Mon. Wea. Rev., 129, 2461-2480.

Eckel, F. A., and C. F. Mass, 2005: Aspects of effective mesoscale, short-range ensemble forecasting. Wea. Forecasting, 20, 328350 .

Fritsch, J. M., and R. E. Carbone, 2004: Improving quantitative precipitation forecasts in the warm season: A USWRP research and development strategy. Bull. Amer. Meteor. Soc., 85, 955-965.

_ J. D. Murphy, and J. S. Kain, 1994: Warm core vortex amplification over land. J. Atmos. Sci., 51, 1780-1807.

Gilmour, I., L. A. Smith, and R. Buizza, 2001: Linear regime duration: Is 24 hours a long time in synoptic weather forecasting? J. Atmos. Sci., 58, 3525-3539.

Hakim, G. J., and R. D. Torn, 2008: Ensemble synoptic analysis. Synoptic-Dynamic Meteorology and Weather Analysis and Forecasting: A Tribute to Fred Sanders, Meteor. Monogr., No. 55, Amer. Meteor. Soc., 147-161.

Hanley, K. E., D. J. Kirshbaum, S. E. Belcher, N. M. Roberts, and G. Leoncini, 2011: Ensemble predictability of an isolated mountain thunderstorm in a high-resolution model. Quart. J. Roy. Meteor. Soc., 137, 2124-2137.

Hawblitzel, D. P., F. Zhang, Z. Meng, and C. A. Davis, 2007: Probabilistic evaluation of the dynamics and predictability of 
the mesoscale convective vortex of 10-13 June 2003. Mon. Wea. Rev., 135, 1544-1563.

Hohenegger, C., A. Walser, W. Langhans, and C. Schär, 2008: Cloud-resolving ensemble simulations of the August 2005 Alpine flood. Quart. J. Roy. Meteor. Soc., 134, 889-904.

Hong, S.-Y., and J.-O. J. Lim, 2006: The WRF single-moment 6-class microphysics scheme (WSM6). J. Korean Meteor. Soc., 42, 129-151.

Hopper, L. J., and C. Schumacher, 2012: Modeled and observed variations in storm divergence and stratiform rain production in southeastern Texas. J. Atmos. Sci., 69, 1159-1181.

Hu, X.-M., J. W. Nielsen-Gammon, and F. Zhang, 2010: Evaluation of three planetary boundary layer schemes in the WRF model. J. Appl. Meteor. Climatol., 49, 1831-1844.

Janjić, Z., 2002: Nonsingular implementation of the MellorYamada level 2.5 scheme in the NCEP meso model. NCEP Office Note 437, 61 pp.

Jankov, I., W. A. Gallus Jr., M. Segal, B. Shaw, and S. E. Koch, 2005: The impact of different WRF model physical parameterizations and their interactions on warm season MCS rainfall. Wea. Forecasting, 20, 1048-1060.

Johns, R. H., and C. A. Doswell III, 1992: Severe local storms forecasting. Wea. Forecasting, 7, 588-612.

Johnson, A., X. Wang, F. Kong, and M. Xue, 2011: Hierarchical cluster analysis of a convection-allowing ensemble during the Hazardous Weather Testbed 2009 spring experiment. Part I: Development of the object-oriented cluster analysis method for precipitation fields. Mon. Wea. Rev., 139, 3673-3693.

Koch, S. E., M. desJardins, and P. J. Kocin, 1983: An interactive Barnes objective map analysis scheme for use with satellite and conventional data. J. Climate Appl. Meteor., 22, 1487-1503.

Leoncini, G., R. S. Plant, S. L. Gray, and P. A. Clark, 2013: Ensemble forecasts of a flood-producing storm: Comparison of the influence of model-state perturbations and parameter modifications. Quart. J. Roy. Meteor. Soc., 139, 198-211.

Lim, K.-S. S., and S.-Y. Hong, 2010: Development of an effective double-moment cloud microphysics scheme with prognostic cloud condensation nuclei $(\mathrm{CCN})$ for weather and climate models. Mon. Wea. Rev., 138, 1587-1612.

Lin, Y., and K. E. Mitchell, 2005: The NCEP stage II/IV hourly precipitation analyses: Development and applications. Preprints, 19th Conf. on Hydrology, San Diego, CA, Amer. Meteor. Soc., P1.2. [Available online at http://ams.confex. com/ams/pdfpapers/83847.pdf.]

Martin, W. J., and M. Xue, 2006: Sensitivity analysis of convection of the 24 May 2002 IHOP case using very large ensembles. Mon. Wea. Rev., 134, 192-207.

Mellor, G. L., and T. Yamada, 1982: Development of a turbulence closure model for geophysical fluid problems. Rev. Geophys., 20, 851-875.

Mesinger, F., and Coauthors, 2006: North American Regional Reanalysis. Bull. Amer. Meteor. Soc., 87, 343-360.

Morrison, H., J. A. Curry, and V. I. Khvorostyanov, 2005: A new double-moment microphysics parameterization for application in cloud and climate models. Part I: Description. J. Atmos. Sci., 62, 1665-1677.

Nakanishi, M., and H. Niino, 2006: An improved Mellor-Yamada level-3 model: Its numerical stability and application to a regional prediction of advection fog. Bound.-Layer Meteor., 119, 397-407.

Nielsen-Gammon, J. W., F. Zhang, A. M. Odins, and B. Myoung, 2005: Extreme rainfall in Texas: Patterns and predictability. Phys. Geogr., 26, 340-364.
NOAA, cited 2012: Storm events database. [Available online at http://www.ncdc.noaa.gov/stormevents/.]

Noh, Y., W. G. Cheon, S. Y. Hong, and S. Raasch, 2003: Improvement of the K-profile model for the planetary boundary layer based on large eddy simulation data. Bound.-Layer Meteor., 107, 401-427.

Novak, D. R., C. Bailey, K. Brill, M. Eckert, D. Petersen, R. Rausch, and M. Schichtel, 2011: Human improvement to numerical weather prediction at the Hydrometeorological Prediction Center. Extended Abstracts, 24th Conf. on Weather Analysis and Forecasting/20th Conf. on Numerical Weather Prediction, Seattle, WA, Amer. Meteor. Soc., P330. [Available online at https://ams.confex.com/ams/91Annual/ webprogram/Manuscript/Paper181989/HumanNWP_final. pdf.]

Raymond, D. J., and H. Jiang, 1990: A theory for long-lived mesoscale convective systems. J. Atmos. Sci., 47, 3067-3077.

Reinecke, P. A., and D. R. Durran, 2009: Initial-condition sensitivities and the predictability of downslope winds. J. Atmos. Sci., 66, 3401-3418.

Rogers, E., and Coauthors, 2009: The NCEP North American mesoscale modeling system: Recent changes and future plans. Extended Abstracts, 23rd Conf. on Weather Analysis and Forecasting/19th Conf. on Numerical Weather Prediction, Omaha, NE, Amer. Meteor. Soc., P2A.4. [Available online at http://ams.confex.com/ams/pdfpapers/154114.pdf.]

Schumacher, R. S., 2011: Ensemble-based analysis of factors leading to the development of a multiday warm-season heavy rain event. Mon. Wea. Rev., 139, 3016-3035.

_ and R. H. Johnson, 2008: Mesoscale processes contributing to extreme rainfall in a midlatitude warm-season flash flood. Mon. Wea. Rev., 136, 3964-3986.

— , and —_, 2009: Quasi-stationary, extreme-rain-producing convective systems associated with midlevel cyclonic circulations. Wea. Forecasting, 24, 555-574.

Schwartz, C. S., and Coauthors, 2010: Toward improved convectionallowing ensembles: Model physics sensitivities and optimizing probabilistic guidance with small ensemble membership. Wea. Forecasting, 25, 263-280.

Sippel, J. A., and F. Zhang, 2008: A probabilistic analysis of the dynamics and predictability of tropical cyclogenesis. J. Atmos. Sci., 65, 3440-3459.

$\longrightarrow$, and - 2010: Factors affecting the predictability of Hurricane Humberto (2007). J. Atmos. Sci., 67, 1759-1778.

Skamarock, W. C., and Coauthors, 2008: A description of the Advanced Research WRF version 3. NCAR Tech. Note NCAR/TN-475+STR, 113 pp. [Available online at http:// www.mmm.ucar.edu/wrf/users/docs/arw_v3.pdf.]

Smirnova, T. G., J. M. Brown, S. G. Benjamin, and D. Kim, 2000: Parameterization of cold-season processes in the MAPS landsurface scheme. J. Geophys. Res., 105 (D3), 4077-4086.

Stensrud, D. J., J.-W. Bao, and T. T. Warner, 2000: Using initial condition and model physics perturbations in short-range ensemble simulations of mesoscale convective systems. Mon. Wea. Rev., 128, 2077-2107.

Storm, B., J. Dudhia, S. Basu, A. Swift, and I. Giammanco, 2009: Evaluation of the Weather Research and Forecasting model on forecasting low-level jets: Implications for wind energy. Wind Energy, 12, 81-90.

Stull, R. B., 1988: An Introduction to Boundary Layer Meteorology. Kluwer Academic, 684 pp.

Sukoriansky, S., B. Galperin, and V. Perov, 2006: A quasi-normal scale elimination model of turbulence and its application 
to stably stratified flows. Nonlinear Processes Geophys., 13, 9-22.

Thompson, G., P. R. Field, R. M. Rasmussen, and W. D. Hall, 2008: Explicit forecasts of winter precipitation using an improved bulk microphysics scheme. Part II: Implementation of a new snow parameterization. Mon. Wea. Rev., 136, 5095-5115.

Torn, R. D., 2010: Diagnosis of the downstream ridging associated with extratropical transition using short-term ensemble forecasts. J. Atmos. Sci., 67, 817-833.

Toth, Z., and E. Kalnay, 1997: Ensemble forecasting at NCEP and the breeding method. Mon. Wea. Rev., 125, 3297-3319.

Trier, S. B., and C. A. Davis, 2002: Influence of balanced motions on heavy precipitation within a long-lived convectively generated vortex. Mon. Wea. Rev., 130, 877-899.

$\longrightarrow$, _, and J. D. Tuttle, 2000a: Long-lived mesoconvective vortices and their environment. Part I: Observations from the central United States during the 1998 warm season. Mon. Wea. Rev., 128, 3376-3395.

— - _ and W. C. Skamarock, 2000b: Long-lived mesoconvective vortices and their environment. Part II: Induced thermodynamic destabilization in idealized simulations. Mon. Wea. Rev., 128, 3396-3412.

Vié, B., O. Nuissier, and V. Ducrocq, 2011: Cloud-resolving ensemble simulations of Mediterranean heavy precipitating events: Uncertainty on initial conditions and lateral boundary conditions. Mon. Wea. Rev., 139, 403-423.

Whiteman, C. D., X. Bian, and S. Zhong, 1997: Low-level jet climatology from enhanced rawinsonde observations at a site in the southern Great Plains. J. Appl. Meteor., 36, 1363-1376.

Xue, M., D. Wang, J. Gao, K. Brewster, and K. K. Droegemeier, 2003: The Advanced Regional Prediction System (ARPS), storm-scale numerical weather prediction and data assimilation. Meteor. Atmos. Phys., 82, 139-170.
— , and Coauthors, 2007: CAPS real-time storm-scale ensemble and high-resolution forecasts as part of the NOAA Hazardous Weather Testbed 2007 spring experiment. Extended Abstracts, 22nd Conf. on Weather Analysis and Forecasting/18th Conf. on Numerical Weather Prediction, Park City, UT, Amer. Meteor. Soc., 3B.1. [Available online at http://ams.confex.com/ams/ pdfpapers/124587.pdf.]

- and Coauthors, 2010: CAPS realtime storm scale ensemble and high resolution forecasts for the NOAA Hazardous Weather Testbed 2010 spring experiment. Extended Abstracts, 25th Conf. on Severe Local Storms, Denver, CO, Amer. Meteor. Soc., 7B.3. [Available online at https://ams.confex.com/ams/ pdfpapers/176056.pdf.]

, and Coauthors, 2011: Realtime convection-permitting ensemble and convection-resolving deterministic forecasts of CAPS for the Hazardous Weather Testbed 2010 spring experiment. Extended Abstracts, 25th Conf. on Weather Analysis and Forecasting/20th Conf. on Numerical Weather Prediction, Seattle, WA, Amer. Meteor. Soc., 9A.2. [Available online at https://ams.confex.com/ams/91Annual/webprogram/ Manuscript/Paper183227/Xue_CAPS_2011_SpringExperiment_ 24thWAF20thNWP_ExtendedAbstract.pdf.]

Zhang, D.-L., and J. M. Fritsch, 1987: Numerical simulation of the meso- $\beta$ scale structure and evolution of the 1977 Johnstown flood. Part II: Inertially stable warm-core vortex and the mesoscale convective complex. J. Atmos. Sci., 44, 2593-2612.

Zhang, F., C. Snyder, and R. Rotunno, 2003: Effects of moist convection on mesoscale predictability. J. Atmos. Sci., 60, 11731185.

Zhang, J., and Coauthors, 2011: National Mosaic and Multisensor QPE (NMQ) system. Bull. Amer. Meteor. Soc., 92, 1321-1338. 